\title{
Photoinduced Millisecond Switching Kinetics in the GFPMut2 E222Q Mutant
}

\author{
Valentina Quercioli, Chiara Bosisio, Stefano C. Daglio, Francesco Rocca, Laura D'Alfonso, \\ Maddalena Collini, Giancarlo Baldini, and Giuseppe Chirico* \\ Dipartimento di Fisica, Università degli Studi di Milano-Bicocca, Piazza della Scienza 3, 20126 Milano, Italy
}

\author{
Stefano Bettati, ${ }^{\ddagger}$ Samanta Raboni, ${ }^{\S}$ and Barbara Campanini \\ Dipartimento di Biochimica e Biologia Molecolare, Università degli Studi di Parma, Viale Usberti 23/A, \\ 43100 Parma, Italy
}

Received: October 21, 2009; Revised Manuscript Received: February 19, 2010

\begin{abstract}
New probes for kinetic intracellular measurements in the millisecond range are desirable to monitor protein biochemical dynamics essential for catalysis, allosteric regulation, and signaling. Good candidates to this aim are the photoswitchable mutants of the green fluorescent protein, whose anionic fluorescence, primed by blue light, is markedly enhanced under an additional excitation at a shorter wavelength and relaxes within a few milliseconds. The aim of this report is to study how the brightness enhancement kinetics depends on the physical-chemical and spectroscopic parameters and to provide proof-of-concept experiments for the use of the fluorescence enhancement in conditions in which the protein diffusion is hindered and thereby photobleaching can be a limiting critical issue. Future, direct applications of photochromic mutants for modulated excitation imaging would in fact require such a detailed knowledge. We present here an extensive study of the photoswitching mechanism of the E222Q mutant of GFPMut2 (Mut2Q), pumped by visible $488 \mathrm{~nm}$ light and probed at 400-420 $\mathrm{nm}$, as a function of $\mathrm{pH}$, viscosity, temperature, and light intensity. In solution, two characteristic photoswitching times are found by means of modulated double beam fluorescence correlation spectroscopy in the 1-30 ms range, depending on the solution $\mathrm{pH}$. The photoswitching kinetics is solved in terms of the eigenvalues and the eigenvectors of a specific energy diagram and used directly to fit the data, suggesting that the observed photoswitching amplitudes and kinetics are related to a single three-level transition loop. Finally, we give in vitro examples of the use of modulated excitation microscopy, based on fluorescence enhancement amplitude and kinetics detection, on Mut2Q protein samples immobilized in acrylamide gels.
\end{abstract}

\section{Introduction}

GFPs from the jellyfish Aequorea victoria and other autofluorescent proteins are generally used as intracellular probes fused with other proteins of interest. ${ }^{1,2}$ The quest to localize proteins and to follow their activity in vivo with higher and higher spatial and time resolution ${ }^{3}$ has motivated the growth of dynamic microspectroscopic techniques, such as fluorescence recovery after photobleaching, and the proposal ${ }^{4}$ and the application $^{5-9}$ of photoactivatable fluorescent proteins to nanoscopy, also under two-photon excitation. ${ }^{10}$ In the latter case, one exploits the selective bleaching and reactivation of GFP proteins to induce sparsely labeled samples as in $\mathrm{PALM}^{5,8}$ or a marked shift of the protein emission to decrease the effective width of the fluorescence point spread function. ${ }^{3,9,11}$ In these and similar cases, one uses photodynamics to enhance the spatial resolution. However, the switching or shifting phenomena are endowed with characteristic response times that can be exploited for dynamic studies. Indeed, a gap seems to exist in the use of GFPderived proteins for the investigation of cellular dynamics on a time scale, 1-10 ms, relevant for dynamic events that are fundamental for protein function and regulation. ${ }^{12}$ With this

* To whom correspondence should be addressed. E-mail: giuseppe.chirico@mib.infn.it. Tel: +39-02-64482440. Fax: +39-0264482585.

* Also part of the Istituto Nazionale di Biostrutture e Biosistemi.

$\S$ Present address: Centro Ricerca Interdipartimentale Biotecnologie Innovative, Università degli Studi di Padova, Viale Colombo 3, 35121, Padova, Italy. regard, the use of modulated techniques seems to be very promising, especially if coupled to imaging methods, such as the recently introduced optical lock-in detection (OLID) imaging microscopy. ${ }^{13}$ Specific mutations in the GFP molecule can provide suitable probes for the application of these methods in the millisecond time range, and part of this report is devoted to the discussion of proof-of-concept experiments for possible applications in this field. It is important to notice that any direct intracellular application of such methods is based on a detailed knowledge of the protein photodynamics in vitro and its dependence on the relevant physical and chemical parameters, at least solution $\mathrm{pH}$, temperature, and viscosity.

The fluorescence emission of most GFP mutants displays a complex photodynamics due to transitions between three levels related to a neutral, an anionic, and a zwitterionic form of the chromophore. The dynamics of the transitions among these states has been found to be in the $50-500 \mu$ s range at basic $\mathrm{pH}$, where the GFP chromophore is mostly deprotonated. ${ }^{14-16}$ The emission of a variety of GFP mutants can also be photoactivated $^{9,17,18}$ by irradiation with blue or UV light ${ }^{3}$ or quenched selectively by stimulated emission with red/infrared radiation. ${ }^{19}$ The photoactivation of these GFP mutants can be almost permanent, ${ }^{9}$ and in some cases, the photoinduced anionic-neutral transition can be reversed by further irradiation. ${ }^{18,20}$ Other GFP mutants, specifically those containing the E222Q mutation, show spontaneous and reversible photoinduced switching events upon irradiation with $410-420 \mathrm{~nm}$ 
laser light. ${ }^{21,22}$ Their photoactivation dynamics, which lies in the millisecond-submillisecond range, ${ }^{21,22}$ deserves deeper investigations due to its high potential value as a tool for monitoring a variety of intracellular processes in a time window, 1-10 ms, typical of many enzymatic reactions $\mathrm{s}^{23}$ and for investigating and monitoring intracellular physical and chemical properties such as temperature, ${ }^{24}$ redox potentials, ${ }^{25}$ or $\mathrm{pH} .{ }^{26}$

The mutation of the glutamate in position 222 into glutamine seems to play a key role in the onset of photochromic properties of GFP. ${ }^{27,28}$ Jung et al. have characterized the effects of this mutation on wild-type GFP and its T203 V mutant in terms of brightness enhancement ${ }^{22,29}$ and estimated the significant relaxation rates from the analysis of the dark and activated state populations at basic $\mathrm{pH}^{22,30}$ The rationalization of the photoactivation mechanism for these mutants is generally based on a four-level model ${ }^{21}$ that involves also the neutral ground state of the chromophore.

In this report, we want to address the possibility of exploiting the GFP photoswitching effect for modulated excitation microscopy. Our point of view is related to the possibility of modulating GFP green emission with a tiny amount of higherenergy (blue) laser radiation that does not induce a dramatic photobleaching even on immobilized proteins and to measuring the fluorescence enhancement and its activation kinetics on each pixel of a confocal image. To this regard, we study the photoswitching amplitude and kinetics with millisecond resolution in solution and on GFPs immobilized in acrylamide gels.

We focus here on the E222Q mutant of GFPMut2 (S65A, V68L, S72A GFP), called hereafter Mut2Q for two main reasons; GPFMut2 is a bright, thoroughly characterized, ${ }^{31-33}$ folding mutant of GFP that can be efficiently expressed in eukaryotic cells and suffers limited photobleaching, ${ }^{34}$ and the mutation S65A stabilizes the deprotonated brighter form at physiological conditions. Moreover, the E222Q mutant of GFPMut2 has particularly low fluorescence efficiency, ${ }^{14}$ thereby offering the possibility of obtaining a large relative fluorescence gain under two-color excitation. The photochromicity of its E222Q mutant, and particularly its characteristic activation time, has not been studied up to now, and this is now covered by this report, whose outline is as follows. We first discuss solution measurements of the photochromic effect in Mut2Q obtained by performing dual-wavelength (pump and probe beams) fluorescence spectroscopy. The dependence of the amplitude of the fluorescence enhancement induced by the probe beam is followed as a function of the (pump/probe) laser intensities, of the probe wavelength, and of the solution $\mathrm{pH}$. We then perform a thorough characterization of the photoswitching times as a function of the relevant physical and chemical parameters, solution temperature, viscosity, and $\mathrm{pH}$, and photoactivation intensity and energy. The switching dynamics and the enhancement amplitudes are measured in solution by devising auto- and cross-correlative methods that can be also applied to imaging microscopy, as shown here in proof-of-concept experiments on Mut2Q trapped in acrylamide gels. After reporting the photophysical characterization in solutions, we propose a minimal energy diagram model whose steady-state and time-dependent solutions are developed in detail in the Supporting Information (SI). In the main text, we discuss how the use of this simple though comprehensive energy diagram model allows one to rationalize the observed double relaxation enhancement dynamics in terms of a single three-level loop. The data analysis is performed by keeping to a minimum the mathematical details that can be found in the SI. Finally, we apply and discuss an imaging protocol that is based on the knowledge of the protein photochromic parameters reported here. This imaging method, named here beating mode imaging, is tested on acrylamide gels loaded with Mut2Q photochromic proteins. The analysis that we apply to the images acquired in the beating mode allows one to envision future intracellular applications, subject to validation experiments not presented here, in which the photoswitching will be a valuable tool for the study of dynamic processes and for the improvement of the image signal-to-noise ratio, similarly to that made in OLID $^{13}$ microscopy.

\section{Experimental Methods}

Protein Expression and Purification and Sample Preparation. GFPmut2 (S65A, V68L, S72A GFP) and its E222Q mutant (Mut2Q) were expressed and purified as described elsewhere. ${ }^{31}$ GFPMut2 is a triple mutant of wild-type Aequorea victoria GFP that ensures high fluorescence stability ${ }^{31-34}$ and high production yield at $37{ }^{\circ} \mathrm{C}$ in prokaryotes..$^{35}$ The samples used for spectroscopic experiments were in $10 \mathrm{mM}$ citrate and $100 \mathrm{mM}$ phosphate buffer solutions, adjusted at the desired $\mathrm{pH}$. All of the measurements were performed at the same buffer concentration since this is known to affect the GFP photodynamics. ${ }^{36}$ The acrylamide gels were prepared at $10 \%(\mathrm{w} / \mathrm{v})$ by diluting an acrylamide-bisacrylamide solution (37.5:1, 40\%, Sigma Aldrich, U.S.A., A7168) with phosphate buffer $\mathrm{pH}=7.8$. The GFP concentration in the gels was between 100 and $150 \mathrm{nM}$.

Optical Setup. The microspectrofluorimeter is based on a Nikon (Japan) TE300 microscope. Two laser sources were employed for two-color fluorescence correlation spectroscopy measurements. A mode-locked Ti:Sapphire laser (Tsunami 3960, Spectra Physics, CA, 280 fs pulse width on the sample, 37,38 repetition frequency $80 \mathrm{MHz}, 700-1000 \mathrm{~nm}$ ) was doubled in order to obtain pulsed laser light in the range of $370-450 \mathrm{~nm}$. A CW argon laser (Spectra Physics, CA, 2025) provided the excitation light at $488 \mathrm{~nm}$ to prime the one-photon absorption of the anionic state of GFP mutants. The confocal acquisition of the signal was done via an SPCM-AQ R15 SPAD module (EG\&G, U.S.A.) and by inserting an $80 \mu \mathrm{m}$ pinhole in the collection optical path. For details, we refer to the SI.

The confocal images were acquired on the same microscope used for solution experiments, equipped with a Plan Apochromat $60 \times$ water objective ( $\mathrm{NA}=1.2$, Nikon, Japan). The sample raster scanning was performed by moving it through a piezo actuator (P-541.2CD, Physik Instrumente, D) with $10 \mathrm{~nm}$ repeatability and $100 \mu \mathrm{m}$ full length displacement in the closed loop mode. The detection of the photon counts and the bias to the three channels of the piezo actuator was performed via an NI analog-digital board and through Lab-view homemade software. ${ }^{38}$

Analysis of the ACFs. The normalized ACFs, acquired in a pseudocrosscorrelation mode, were computed by an ALV5000E (ALV, Langen, Germany) board and analyzed by means of the non-least-square routine of the Origin 7.0 software (OriginLab Inc., Northampton, MA). The ACF decay was analyzed according to the model function ${ }^{15}$

$$
\begin{aligned}
& G(t)=\frac{0.35}{\langle N\rangle}\left(1+\frac{t}{\tau_{\mathrm{D}}}\right)^{-1}\left(1+\frac{t}{\tau_{\mathrm{z}}}\right)^{-0.5} \times \\
& \prod_{j}\left[1+\frac{A_{j}}{1-A_{j}} \exp \left(-t / \tau_{j}\right)\right]
\end{aligned}
$$

where $A_{j}$ and $\tau_{j}$ are the fraction of the $j$ th dark species and the corresponding bright $\rightarrow$ dark transition relaxation time, $\langle N\rangle$ is the average number of observed molecules endowed with 
diffusion coefficient $D$, and $\tau_{\mathrm{D}}$ and $\tau_{\mathrm{z}}$ are the radial and axial diffusion times, $\tau_{\mathrm{D}}=\omega_{0}^{2} / 4 D$ and $\tau_{\mathrm{z}} \cong 5 \tau_{\mathrm{D}}$. Since the ACFs have been routinely fitted down to $10 \mu \mathrm{s}$, the triplet state dynamics has not been taken into account (see SI for further details).

Analysis of the ACFs under Modulated Excitation. The zero time lag extrapolation of the autocorrelation function $(\mathrm{ACF})$, free of electronic artifacts as measured in the pseudocrosscorrelation mode,${ }^{39}$ is

$$
G(0)=\frac{\langle\delta F(t) \delta F(t)\rangle}{\langle F(t)\rangle^{2}}=\frac{\left\langle F(t)^{2}\right\rangle-\langle F(t)\rangle^{2}}{\langle F(t)\rangle^{2}}
$$

This parameter can be used to obtain information on any relaxation time $\tau$ that characterizes the fluorescence emission dynamics if the excitation intensity is modulated periodically at frequencies close to $1 / \tau$. When the excitation beam is modulated as a harmonic function with angular frequency $\omega$, $I(t)=I_{0}\left(1+A_{1} \sin (\omega t)\right)$, the corresponding fluorescence emission, $F(t)$ is modulated at the same frequency $\omega,{ }^{40}$ but it is demodulated in amplitude and delayed in phase $F(t)=F_{0}(1+$ $A_{1} m(\omega) \sin (\omega t-\phi(\omega))$ if $\omega$ is close to $1 / \tau$. The relevant quantities are the demodulation ratio $m(\omega)$ and the phase shift $\phi(\omega)$ whose forms are $m(\omega)=\left(1+\omega^{2} \tau^{2}\right)^{-1 / 2}$ and $\phi(\omega)=$ arct $g(\omega \tau)$, for a single relaxation time. The form of $m(\omega)$ and $\phi(\omega)$ can be obtained by a Fourier analysis of the fluorescence signal obtained under modulated excitation. The underlying theoretical treatment has been extensively reported in the literature $^{40}$ and applied experimentally in the field of characterization of the molecular excited states that lie in the nanosecond range. In these cases, the excitation light is modulated at hundreds of $\mathrm{MHz}$. Here, we exploit the same treatment in the $\mathrm{Hz}-\mathrm{kHz}$ range (see $\mathrm{SI}$ ).

The zero time extrapolation $G(0)$ of the $\mathrm{ACF}$, measured under an intensity modulated laser beam, can be obtained from eq 2 and the harmonic form $F(t)=F_{0}\left(1+A_{1} m(\omega) \sin (\omega t-\phi(\omega))\right.$ as follows

$$
\left\{\begin{array}{c}
\langle F(t)\rangle^{2}=F_{0}^{2} \\
\left\langle F(t)^{2}\right\rangle=F_{0}^{2}+\frac{F_{0}^{2} A_{1}^{2} m(\omega)^{2}}{2} \\
G(0, \omega)=\frac{\left\langle F(t)^{2}\right\rangle-\langle F(t)\rangle^{2}}{\langle F(t)\rangle^{2}}=A_{1}^{2} \frac{m(\omega)^{2}}{2}
\end{array}\right.
$$

Since the pump beam is always present, a constant nonmodulated signal, $F_{\mathrm{B}}$, must be added to the fluorescence emission, whose average value is $F_{0}$. The average squared fluorescence value increases then to $\left(F_{0}+F_{\mathrm{B}}\right)^{2}$ in eqs 2 and 3 . The fluorescence fluctuations are instead affected only by the average squared value of the correlated fluorescence photons, $F_{0}^{2}$. In practice, $F_{\mathrm{B}}$ is the value of the constant nonmodulated signal due to the second laser beam used for two-color FCS, and the amplitude of the modulation $(G(0))$ is diminished because the oscillations are superimposed on a background. Equation 3 changes then to the final form

$$
G(0, \omega)=A_{1}^{2} \frac{m(\omega)^{2}}{2} \frac{F_{0}^{2}}{\left(F_{0}+F_{\mathrm{B}}\right)^{2}}
$$

For the current case of a square wave modulation, the trend of $G(0, \omega)$ as a function of the modulation frequency can be obtained by Fourier expanding the excitation as $I(t)=I_{0}(1+$ $\left.\sum_{n} A_{2 n+1} \sin ((2 n+1) \omega t)\right)$ and writing eq 4 as a sum of the contributions of the first few harmonic components.

Moreover, when the fluorescence dynamics of the system is endowed with two relaxation times, $\tau_{\mathrm{R} 1}$ and $\tau_{\mathrm{R} 2}$, with fractions $f_{1}$ and $f_{2}, G(0, \omega)$ can be written as (see SI for further details)

$$
\begin{gathered}
G(0, \omega)=\frac{F_{0}^{2}}{\left(F_{0}+F_{\mathrm{B}}\right)^{2}} \frac{1}{2} \sum_{n=0,1,2} A_{2 n+1}^{2} \\
{\left[\left(\frac{f_{1}}{1+(2 n+1)^{2} \omega^{2} \tau_{1}^{2}}+\frac{1-f_{1}}{1+(2 n+1)^{2} \omega^{2} \tau_{2}^{2}}\right)^{2}+\right.} \\
\left.\left(\frac{f_{1}(2 n+1) \omega \tau_{1}}{1+(2 n+1)^{2} \omega^{2} \tau_{1}^{2}}+\frac{\left(1-f_{1}\right)(2 n+1) \omega \tau_{2}}{1+(2 n+1)^{2} \omega^{2} \tau_{2}^{2}}\right)^{2}\right]+\beta
\end{gathered}
$$

The constant background $\beta$ accounts for the presence of any dynamic contribution to the demodulation with relaxation times $\ll \tau_{1}, \tau_{2}$. For the frequency range explored here, more than three terms in the square wave expansion neither improve the quality of the fit nor change the values of the best-fit parameters.

\section{Results and Discussion}

Equilibrium Fluorescence Spectroscopy. The mutation of Ser65 accounts for the $\mathrm{pH}$ dependence of GFPmut2 spectroscopic properties, with a $\mathrm{p} K_{\mathrm{a}}$ for the chromophore anionic-neutral equilibrium of $6.12 \pm 0.01$ and $6.0 \pm 0.1$ for GFPMut2 and Mut2Q, respectively. ${ }^{31}$ The absorption spectra of the two mutants show absorption bands at $\sim 390 \mathrm{~nm}$ for the chromophore neutral state and $\sim 490 \mathrm{~nm}$ for the anionic state, with an isosbestic point at $425 \mathrm{~nm}$ (ref 31 and SI), consistent with an apparent two-state transition. Excitation at $490 \mathrm{~nm}$ leads to fluorescence emission at $508 \mathrm{~nm}$, typical of the anionic form of the chromophore, in a wide range of $\mathrm{pH}$ values. Excitation at $390 \mathrm{~nm}$, instead, does not produce relevant emission at 508 $\mathrm{nm}$ (see SI), indicating that a very small or no excited-state proton transfer (ESPT) is present in Mut2Q due to the E222Q mutation. ${ }^{27,41}$

When Mut2Q is excited simultaneously by a $488 \mathrm{~nm}$ pump beam and a more energetic probe beam at $405 \mathrm{~nm}$, the $508 \mathrm{~nm}$ anionic emission at alkaline $\mathrm{pH}(\mathrm{pH}=8.4)$ is substantially larger than the sum of the emissions primed by one beam at a time (Figure 1A). The almost linear increase of the fluorescence emission as a function of the probe beam intensity observed when the protein solution is irradiated by the probe beam only (Figure $1 \mathrm{~A}$ ) is due to the presence at $\mathrm{pH} \cong 8.4$ of $\cong 80 \%$ of chromophores in the anionic state that absorbs the $405 \mathrm{~nm}$ probe beam about 20 times less than the neutral state. ${ }^{31}$ No appreciable fluorescence enhancement induced by two-color irradiation is found for the GFPMut2 parent protein (Figure 1A, inset), suggesting that the molecular origin of the effect is related to the E222Q mutation, in agreement with Jung et al. ${ }^{28}$ A minor decrease of the emission is actually observed at increasing probe excitation intensity, probably due to some additional photobleaching induced by the more energetic beam irradiation.

The emission spectra under dual beam excitation, recorded as a function of the probe beam intensity by means of a CCD spectrometer (see SI), are peaked at $508 \mathrm{~nm}$, as in the case of single beam excitation, suggesting that the enhancement mechanism is due to emission from the anionic state of the protein.

Effect of the Pump and Probe Beams' Intensity on the Fluorescence Enhancement. The anionic emission increases linearly with excitation intensity and reaches a plateau level at 


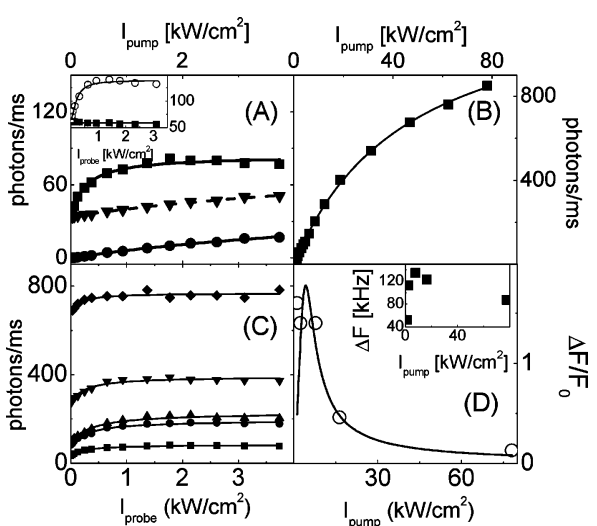

Figure 1. Fluorescence emission $\left(\lambda_{\mathrm{em}}=515 / 30 \mathrm{~nm}\right)$ of Mut2Q at $\mathrm{pH}$ $=8.4$ versus laser intensity. (A) Probe excitation only $(405 \mathrm{~nm}$, filled circles); dual beam excitation (pump at $488 \mathrm{~nm}, I_{\text {pump }}=1.1 \mathrm{~kW} / \mathrm{cm}^{2}$, and probe at $405 \mathrm{~nm}$, filled squares). Dashed line and triangles: fluorescence signal computed in the case of no photoinduced fluorescence enhancement. Solid lines: best fit of eq 6 to the data (Table 1). Inset: fluorescence emission under dual beam excitation versus the probe intensity $\left(I_{\text {pump }}=1.7 \mathrm{~kW} / \mathrm{cm}^{2}\right)$ for GFPMut2 (filled squares) and Mut2Q (open circles). Data refer to the same protein concentration, and the solid lines are as those in the main panel. (B) Saturation curve of the fluorescence emission of Mut2Q at $\mathrm{pH} 8.4$ versus the pump beam ( 488 $\mathrm{nm}$ ) intensity. Solid line: best fit to eq 6 (see Table 1). (C) Fluorescence emission of Mut2Q versus the probe $(405 \mathrm{~nm})$ intensity at different pump intensity values, 1.1 (squares), 2.3 (circles), 7.7 (up triangles), 16.3 (down triangles), and $78 \mathrm{~kW} / \mathrm{cm}^{2}$ (diamonds). Solid lines: best fit of the data to eq 6 (see Table 1). (D) Relative fluorescence enhancement, $\Delta F / F_{0}$, versus the pump intensity derived from the data fitting. Solid line: best fit to eq 11. Inset: absolute fluorescence enhancement, $\Delta F$, versus the pump beam intensity.

TABLE 1: Fluorescence Output As a Function of the Pump or Probe Beam Intensity ${ }^{a}$

\begin{tabular}{lccccc}
\hline & $I_{\text {pump }}$ & $I_{\text {sat }}$ & $\Delta F[\mathrm{kHz}]$ & $F_{0}[\mathrm{kHz}]$ & $\Delta F / F_{0}$ \\
\hline probe only & & $7.8 \pm 1.3$ & $54 \pm 7$ & $-0.2 \pm 2$ & - \\
pump only & & $49 \pm 2$ & $1370 \pm 30$ & - & - \\
pump\&probe & 1.1 & $0.26 \pm 0.04$ & $52 \pm 2$ & $33 \pm 2$ & $1.6 \pm 0.1$ \\
& 2.3 & $0.33 \pm 0.05$ & $113 \pm 4$ & $81 \pm 3$ & $1.4 \pm 0.07$ \\
& 7.7 & $0.39 \pm 0.10$ & $134 \pm 7$ & $95 \pm 6$ & $1.4 \pm 0.1$ \\
& 16.3 & $0.24 \pm 0.06$ & $123 \pm 7$ & $268 \pm 7$ & $0.46 \pm 0.03$ \\
& 78 & $0.16 \pm 0.08$ & $87 \pm 10$ & $683 \pm 10$ & $0.13 \pm 0.01$
\end{tabular}

${ }^{a}$ Result of the analysis of the fluorescence signal as a function of the pump or probe beam. Best fit parameters obtained by fitting eq 6 to the fluorescence increase as a function of the pump or probe beam intensity. For the pump\&probe irradiation, the pump beam intensity was set to $1.1 \mathrm{~kW} / \mathrm{cm}^{2}$. All of the intensity values are reported in $\mathrm{kW} / \mathrm{cm}^{2}$.

high excitation intensity values both under the pump beam only and pump\&probe beam irradiation (Figure 1B,C). This behavior can be described by the function ${ }^{42}$

$$
\langle F\rangle=F_{0}+\Delta F\left(\frac{I}{I_{\mathrm{sat}}+I}\right)
$$

where $F_{0}$ is the fluorescence measured at excitation intensity $I$ $=0$ and $\Delta F$ is the maximum fluorescence increase observed.

When increasing the pump beam intensity under no probe excitation (Figure $1 B$ ), we find the value of the saturation intensity to be $I_{\text {pump,sat }}=49 \pm 3 \mathrm{~kW} / \mathrm{cm}^{2}$, substantially lower than the value at which the ground state becomes depleted due to the finite lifetime, $\tau$, of the excited state, $I_{\text {depletion }} \cong 5000 \mathrm{~kW} /$ $\mathrm{cm}^{2}$. This value can be computed for the ground-state depletion (excited-state lifetime $\tau \cong 1 \mathrm{~ns},{ }^{14}$ one-photon absorption cross

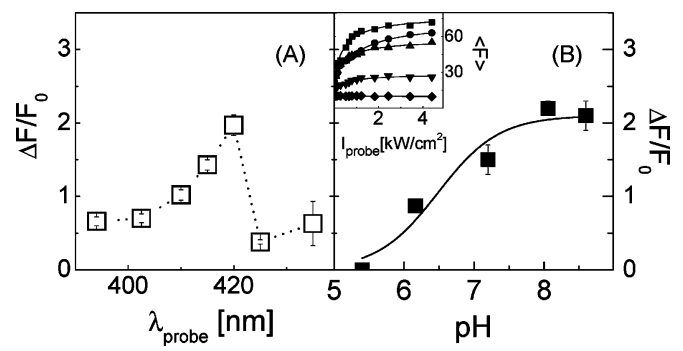

Figure 2. (A) Fluorescence enhancement, $\Delta F / F_{0}$, versus the probe wavelength. (B) Fluorescence enhancement, $\Delta F / F_{0}$, versus the solution $\mathrm{pH}$. The solid line is the best fit of a sigmoidal curve, $y=2.1 /[1+$ $\left.10^{\left(\mathrm{pH}-\mathrm{p} K^{*}\right) / \delta}\right]$, to the $\mathrm{pH}$-dependent data. The best fit parameters are $\mathrm{p} K^{*}$ $=6.5 \pm 0.1$ and $\delta=1.1 \pm 0.13$. The pump beam intensity was 1.7 $\mathrm{kW} / \mathrm{cm}^{2}$. The inset shows the fluorescence emission (raw data in photons $/ \mathrm{ms})$ of Mut $2 \mathrm{Q}$ versus the probe intensity $\left(\lambda_{\text {probe }}=420 \mathrm{~nm}\right)$ at various $\mathrm{pH}$ values of 8.6 (squares), 8.06 (circles), 7.2 (up triangles), 6.2 (down triangles), and 5.4 (diamonds). The solid lines are the best fit to eq 6 .

section $^{27} \sigma_{1} \cong 10^{-16} \mathrm{~cm}^{2}$, photon energy $\left.E_{\mathrm{ph}} \cong 4 \times 10^{-19} \mathrm{~J}\right)$ as $I_{\text {depletion }} \cong E_{\mathrm{ph}} /\left(\tau \sigma_{1}\right)$. Therefore, the saturation may be ascribed to intersystem crossing, a process often invoked to account for the saturation of the fluorescence emission of GFP mutants ${ }^{15}$ and/or the emergence of transitions to higher-energy states. ${ }^{42}$

The trend of the fluorescence emission of Mut2Q as a function of the probe intensity for a wide range of pump beam intensities, $1 \leq I_{\text {pump }} \leq 80 \mathrm{~kW} / \mathrm{cm}^{2}$, is reported in Figure $1 \mathrm{C}$, and the corresponding best-fit parameters are reported in Table 1. Under two-color excitation, the fluorescence emission at increasing probe beam intensity saturates at $I_{\text {probe,sat }} \cong 0.3 \mathrm{~kW} / \mathrm{cm}^{2}$ over almost the whole range of $I_{\text {pump }}$ values investigated. The two fluxes of photons (pump and probe) act independently on the observed emission enhancement, and this can be taken as an indication that the emission increase observed under either pump or probe (actually pump\&probe) excitation originates from priming transitions from different lower-energy molecular states.

The fluorescence enhancement induced by the probe beam, $\Delta F$, shows a maximum (Figure $1 \mathrm{D}$, inset) at a power that is close to the value of the saturating pump intensity (Figure 1B). When $\Delta F$ is normalized to the fluorescence output under pump excitation only, $F_{0}$, it rapidly decays as a function of the pump beam intensity (Figure 1D), indicating that the lower the pump excitation, the larger the relative fluorescence enhancement. The trend of $\Delta F$ follows therefore the trend of the pump-induced emission, and in particular, it decreases above the value of the saturation intensity. We may therefore hypothesize that the complex photodynamic process that leads to the fluorescence enhancement has as a limiting step the excitation of the chromophore to its first anionic (since we are exciting at 488 $\mathrm{nm}$ ) excited state.

Altogether, we come then to a possible scenario in which the pump and probe beams prime two consecutive processes, the first one being the transition of the anionic chromophore to its first excited state. We further hypothesize in the following analysis and in the development of an energy diagram model that the decrease of $\Delta F$ for $I_{\text {pump }} \geq I_{\text {pump,sat }}$ is due to transitions from the first excited state to higher-energy states.

Effect of the Probe Beam Wavelength and Solution pH on Fluorescence Enhancement. The enhancement factor $\Delta F$ / $F_{0}$ depends on the probe beam wavelength in the range of 390-440 nm (Figure 2A), with a smooth increase while raising the photon wavelength up to a maximum effect at $420 \mathrm{~nm}$, followed by a rapid drop at larger wavelengths. This shape is an indication of the presence of a smooth distribution of energy 
state gaps, corresponding to the transition primed by the 420 $\mathrm{nm}$ photons, characterized by a minimum threshold value. In fact, for wavelengths larger than $\lambda=420 \mathrm{~nm}$, that is, for photons with energy smaller than $\Delta E=h \lambda / c \cong 3 \mathrm{eV}$, the transition cannot occur, or it occurs with much lower efficiency. The behavior described above is the fingerprint of a resonant interaction with an energy gap of $\Delta E=h \lambda / c \cong 3 \mathrm{eV}$ ( $h$ is the Planck constant).

The unchanged shape of the emission spectrum under dual beam excitation discussed above indicates that the final step in the observed photochromic behavior of Mut2Q under dual-color excitation is the de-excitation of the anionic state of the chromophore. However, this experimental result does not allow one to exclude some involvement of the neutral state of the chromophore in its photoactivation, as suggested by Jung et al. for E222Q GFP. ${ }^{21}$

We have therefore investigated the fluorescence emission of Mut2Q over a wide range of $\mathrm{pHs}, 5.4 \leq \mathrm{pH} \leq 9$, under dual beam excitation at 488 and $420 \mathrm{~nm}$ (Figure 2B). The dependence of the fluorescence on the probe beam intensity can again be fit by eq 6 . The fluorescence measured under pump excitation only, $F_{0}$, decreases at acidic $\mathrm{pH}$ values (Figure 2) since fewer and fewer molecules lie in the anionic state and can be excited at $488 \mathrm{~nm}$. Also, the fluorescence enhancement, $\Delta F$, decreases at acidic $\mathrm{pH}$ values and almost vanishes at $\mathrm{pH}=5.4$. The enhancement factor, $\Delta F / F_{0}$, varies markedly between acidic and basic conditions, and its $\mathrm{pH}$ dependence (Figure 2 ) is described by a protonation curve with a $\mathrm{p} K_{\mathrm{a}}$ value of $6.5 \pm 0.2$, slightly larger than that measured from the $\mathrm{pH}$ titration of the anionic fluorescence emission, $\mathrm{p} K_{\mathrm{a}}=6.0 \pm 0.1 .{ }^{14}$ We have also verified that at low $\mathrm{pH}$ values, the low enhancement effect cannot be increased by changing the probe wavelength. This suggests that the fluorescence enhancement is directly related to the absorption of the chromophore in the anionic form, with minor, if any, role of the neutral ground state, contrary to what was previously reported for the E222Q mutant of wild-type GFP. ${ }^{22}$ The identity of the state involved in the enhancement effect is not easy to ascertain and would be rather speculative at this stage.

Fluorescence Enhancement Dynamics. Fluorescence correlation spectroscopy (FCS) covers a dynamic range from microseconds to seconds. ${ }^{43}$ Mut2Q photodynamics upon singlephoton excitation at $488 \mathrm{~nm}^{14}$ shows two exponential decays at alkaline $\mathrm{pH}$ values due to a power-dependent triplet conversion (5-20 $\mu \mathrm{s})$ and a light-induced power-dependent photoswitching $(50-200 \mu \mathrm{s})$. For other E222Q mutants, it has been suggested ${ }^{21}$ that the conversion rates among the energy levels involved in the enhancement correspond to a relaxation time, $\tau_{\mathrm{R}}$, in the 10 $\mathrm{ms}$ range that is on the order of the protein dwell times in the excitation volume.

The dynamic component related to the fluorescence enhancement and characterized by the relaxation time $\tau_{\mathrm{R}}$ is singled out under two-color modulated excitation (either of the probe or the pump) in a frequency range centered around the value $v$ $=1 / \tau_{\mathrm{R}}$. This approach is the same as the frequency domain method to measure the excited-state lifetime of fluorophores. ${ }^{40}$ When a two-level quantum system is excited by light whose intensity is modulated at a frequency close to the inverse, $1 / \tau_{\mathrm{R}}$, of the relaxation time between the two states, $\tau_{R}$, the emission is modulated at the same frequency with a demodulation ratio and a phase shift due to the finite value of $\tau_{\mathrm{R}}$ (see Experimental Methods section). By measuring these parameters as a function of the modulation frequency, it is possible to derive $\tau_{\mathrm{R}} \cdot{ }^{40}$ For the measurement of the lifetime of an electronic excited state that is typically of the order of few nanoseconds, one modulates

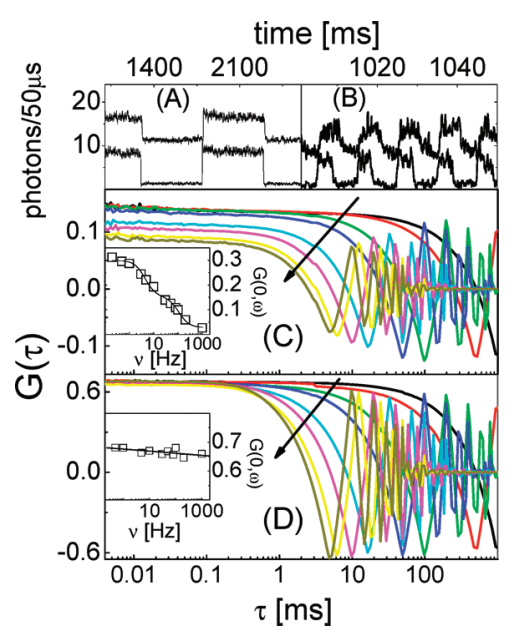

Figure 3. $(\mathrm{A}, \mathrm{B})$ Time traces of the fluorescence emission primed by the $488 \mathrm{~nm}$ pump beam $(20 \mathrm{kHz}$ sampling frequency, smoothed over 15 adjacent points), acquired under either pump or probe beam modulation. $I_{\text {probe }}$ was $1.6 \mathrm{~kW} / \mathrm{cm}^{2}$, and $I_{\text {pump }}$ was $2.3 \mathrm{~kW} / \mathrm{cm}^{2}$. (A) and (B) refer to modulation frequencies of 1 and $100 \mathrm{~Hz}$, respectively. In each panel, the upper curve, displaced for display purposes, refers to the modulation of the probe beam and the lower curve to the pump beam modulation. (C,D) Fluorescence ACFs computed while modulating either the probe (C) or the pump (D) laser beams at increasing modulation frequencies, $v=0.5$ (black), 1 (red), 5 (green), 10 (blue), 30 (cyan), 50 (magenta), 80 (yellow), and $100 \mathrm{~Hz}$ (dark olive). The corresponding zero lag time extrapolation values for $G(0, \omega)$ are reported in the panel insets. The solid line in the (C) inset is the best fit of the probe modulation data to eq 5 . The solid line in the (D) inset is a linear best fit to the data, drawn to guide the eye. The pump and probe excitation intensities were $I_{\text {pump }}=16.3 \mathrm{~kW} / \mathrm{cm}^{2}$ and $I_{\text {probe }}=1.6 \mathrm{~kW} /$ $\mathrm{cm}^{2}$ in all of the experiments.

the excitation intensity at frequencies on the order of 100-400 $\mathrm{MHz}$. We have employed here modulation frequencies in the range of $1-2000 \mathrm{~Hz}$ (since we want to explore relaxation times of $\tau_{\mathrm{R}} \cong 0.5-1000 \mathrm{~ms}$ ) and directly measured the demodulation ratios from the computation of the normalized mean-square fluorescence fluctuation, $G(0)$ (eqs 2 and 5).

The basic idea of this experimental approach is to modulate either the pump or the probe beam excitation intensities and to measure the demodulation and dephasing of the emitted light as a function of the modulation frequency. The modulation frequencies are here slow enough to be obtained by inserting a chopper or an electronic shutter in the beam path, thereby modulating the intensity by a square wave. The frequency of the square wave is then the first harmonic of the frequency content of the emitted light.

The typical time traces of the fluorescence emission acquired while modulating either the pump or the probe beam intensity by a square wave function are shown in Figure 3A (frequency modulation of $1 \mathrm{~Hz}$ ) and Figure 3B (frequency modulation of $100 \mathrm{~Hz}$ ). The stepwise change of the square wave excitation is systematically rounded off in the emission when the probe beam is modulated at $100 \mathrm{~Hz}$, if compared to lower frequencies (Figure 3A,B upper curves), whereas no low-pass filtering effect on the fluorescence trace is present upon modulation of the pump beam (Figure 3A,B lower curves). This effect can be quantified by the computation of the fluorescence fluctuation ACFs. This function shows marked peaks at times corresponding to the applied modulation period (Figure 3C,D) and varying meansquare fluorescence fluctuation amplitudes. When modulating the pump beam (Figure 3D), small changes are observed in the zero time delay extrapolation of the ACF, $G(0, \omega)$, as shown in the inset of Figure 3D. On the contrary, a strong dependence 


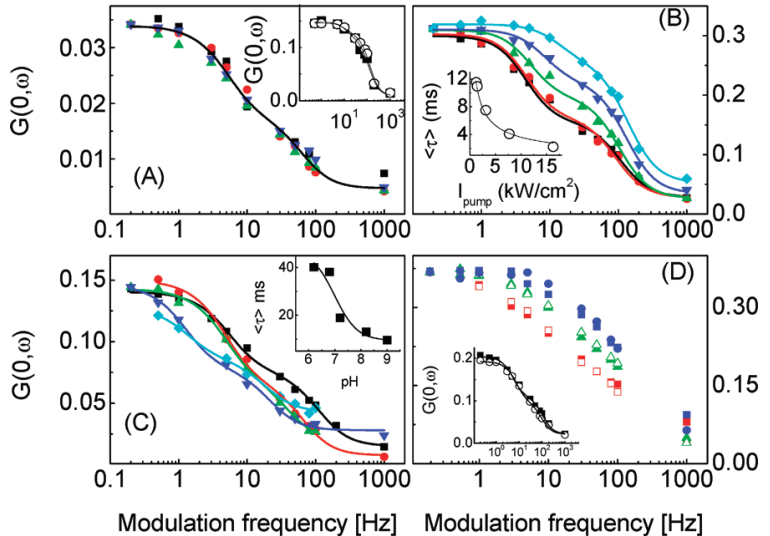

Figure 4. Dependence of the demodulation factor, $G(0, \omega)$, on experimental parameters. (A) Trend of $G(0, \omega)$ versus the modulation frequency for probe excitation intensities of 0.12 (squares, black), 0.31 (circles, red), 0.51 (up triangles, green), and $0.93 \mathrm{~kW} / \mathrm{cm}^{2}$ (down triangles, blue) on the sample. The solid line is the global fitting of the data to eq 5. Inset: $G(0, \omega)$ dependence on the probe wavelength; the filled squares and open circles refer to the modulated probe excitation at 420 and $403 \mathrm{~nm}$, respectively. (B) Trend of $G(0, \omega)$ versus the modulation frequency for pump excitation intensities of 1.2 (squares, black), 1.4 (circles, red), 3.1 (up triangles, green), 7.7 (down triangles, blue), and $16.3 \mathrm{~kW} / \mathrm{cm}^{2}$ (diamonds, cyan). The solid lines are the best fit to eq 5 (see Table 2). The inset reports the average relaxation time as a function of $I_{\text {pump. }}$. The line is the best fit of eq $12\left(\tau_{1}\right)$ to the data. (C) Demodulation factor versus the modulation frequency at various $\mathrm{pH}$ values, 6.2 (diamonds, cyan), 6.8 (down triangles, blue), 7.2 (up triangles, green), 8.2 (circles, red), and 9 (squares, black). Solid lines are the best fit of eq 5 to the data (Table 3 ). The inset reports the average relaxation time versus $\mathrm{pH}$; the solid line is a sigmoidal fit yielding a $\mathrm{p} K_{\mathrm{a}}=7.0 \pm 0.1$. (D) $G(0, \omega)$ versus modulation frequency for $I_{\text {probe }}=$ $1.35 \mathrm{~kW} / \mathrm{cm}^{2}$ and $I_{\text {pump }}=0.8$ (squares, blue), 8.0 (triangles, green), and $15.6 \mathrm{~kW} / \mathrm{cm}^{2}$ (circles, red). The filled and open symbols refer to solution temperatures of 24 and $38^{\circ} \mathrm{C}$, respectively. Inset: $G(0, \omega)$ versus the solution viscosity, changed by adding glycerol. The filled and open symbols refer to 0 and $50 \%$ glycerol, respectively.

of the $G(0, \omega)$ value on the modulation frequency is found when the probe beam is modulated (inset of Figure $3 \mathrm{C}$ ). This is in agreement with the direct observations of Figures $3 \mathrm{~A}$ and $\mathrm{B}$.

The analysis of the modulation factor, $G(0, \omega)$, as a function of the modulation frequency, $v=\omega / 2 \pi$, can be performed according to conventional Fourier methods, ${ }^{40}$ as described in the Experimental Methods section. The modulation of the pump laser beam induces only a minor decrease of $G(0, \omega)$ (Figure $3 \mathrm{D})$, consistent with the traces reported in Figure $3 \mathrm{~A}, \mathrm{~B}$, while the modulation of the probe beam affects substantially $G(0, \omega)$ (Figure 3C), which decreases steadily with the modulation frequency. The step-like behavior observed in the inset of Figure $3 \mathrm{C}$ suggests that a single characteristic time does not adequately describe the data, which can instead be fitted by an equation with two relaxations (eq 5 and SI) providing characteristic times of $\tau_{1}=1.3 \pm 0.2$ and $\tau_{2}=32.8 \pm 4.7 \mathrm{~ms}$, with fractional weight $f_{1}=68 \pm 4 \%$ at $\mathrm{pH} \cong 8.5$ and $I_{\text {pump }}=1.7 \mathrm{~kW} / \mathrm{cm}^{2}$.

Effect of the Pump and Probe Intensity on the Enhancement Dynamics. We have acquired probe-modulated FCS curves at 403 and $420 \mathrm{~nm}$ and found almost no difference within the experimental error (Figure 4A and Table 2). A limited effect is also found when changing the probe intensity in the range of $0.05 \leq I_{\text {probe }} \leq 1 \mathrm{~kW} / \mathrm{cm}^{2}$, with $I_{\text {pump }}=1 \mathrm{~kW} / \mathrm{cm}^{2}$ and $\mathrm{pH}=$ 8.2 (Figure 4A). A global fit of the whole set of data curves provides the best-fit parameters $\tau_{1}=2.4 \pm 0.2 \mathrm{~ms}, \tau_{2}=29 \pm$ $2 \mathrm{~ms}$, and $\mathrm{f}_{l}=65 \pm 4 \%$.

On the contrary, the rise of the pump beam intensity leads to an increase of the demodulation factor $G(0, \omega)$ (Figure 4B, Table
TABLE 2: Dependence of $G(0, \omega)$ on the Pump or Probe Laser Beam Intensity ${ }^{a}$

\begin{tabular}{|c|c|c|c|c|}
\hline & $f_{1}$ & $\tau_{1, \mathrm{R}}[\mathrm{ms}]$ & $\tau_{2, \mathrm{R}}[\mathrm{ms}]$ & $\langle\tau\rangle[\mathrm{ms}]$ \\
\hline$\lambda[\mathrm{nm}]$ & \multicolumn{4}{|c|}{$I_{\text {pump }}=17.0 \mathrm{~kW} / \mathrm{cm}^{2}$} \\
\hline 405 & $0.90 \pm 0.05$ & $1.3 \pm 0.2$ & $12.9 \pm 5.0$ & $2.4 \pm 0.6$ \\
\hline 420 & $0.94 \pm 0.04$ & $1.2 \pm 0.2$ & $14.9 \pm 7.3$ & $2.0 \pm 0.6$ \\
\hline$I_{\text {pump }}$ & \multicolumn{4}{|c|}{$I_{\text {probe }}=0.93 \mathrm{~kW} / \mathrm{cm}^{2}$} \\
\hline 1.7 & $0.68 \pm 0.02$ & $1.3 \pm 0.2$ & $32.8 \pm 4.7$ & $11.5 \pm 2.4$ \\
\hline 2.1 & $0.69 \pm 0.03$ & & $31.8 \pm 2.8$ & $11.0 \pm 2.0$ \\
\hline 3.8 & $0.77 \pm 0.02$ & & $28.3 \pm 4.4$ & $7.5 \pm 1.6$ \\
\hline 8.5 & $0.83 \pm 0.02$ & & $18.7 \pm 3.5$ & $4.1 \pm 1.0$ \\
\hline 17.0 & $0.89 \pm 0.03$ & & $11.1 \pm 5.3$ & $2.2 \pm 0.6$ \\
\hline
\end{tabular}

${ }^{a}$ Analysis of the $G(0, \omega)$ dependence on the laser intensity. The best fit parameters were obtained by fitting eq 5 to the probe modulation data reported in Figures 3 and 4.

TABLE 3: Dependence of $G(0, \omega)$ on the Modulation Frequency at Different pH Values ${ }^{a}$

\begin{tabular}{cccccc}
\hline $\mathrm{pH}$ & $f_{1}$ & $\tau_{1, \mathrm{R}}[\mathrm{ms}]$ & $\tau_{2, \mathrm{R}}[\mathrm{ms}]$ & $\beta$ & $\langle\tau\rangle[\mathrm{ms}]$ \\
\hline \multicolumn{5}{c}{$I_{\text {pump }}=1.7 \mathrm{~kW} / \mathrm{cm}^{2}$} \\
\end{tabular}

$\begin{array}{llllll}6.2 & 0.70 \pm 0.04 & 8.2 \pm 2.0 & 113.9 \pm 44 & 0.042 \pm 0.003 & 40.1 \pm 14.0\end{array}$ $\begin{array}{lllllll}6.8 & 0.65 \pm 0.03 & 6.7 \pm 1.4 & 94.7 \pm 30 & 0.027 \pm 0.002 & 38.1 \pm 10.9\end{array}$ $\begin{array}{llllll}7.2 & 0.61 \pm 0.07 & 3.9 \pm 1.5 & 32.5 \pm 8.5 & 0.019 \pm 0.006 & 18.8 \pm 4.1\end{array}$ $\begin{array}{llllll}8.2 & 0.64 \pm 0.05 & 2.3 \pm 0.2 & 31.7 \pm 7.0 & 0.007 \pm 0.004 & 13.0 \pm 3.0\end{array}$

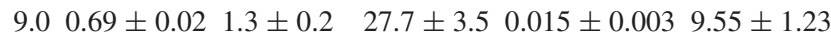

${ }^{a}$ Analysis of the $G(0, \omega)$ dependence on the modulation frequency at different $\mathrm{pH}$ values. The best fit parameters were obtained by analyzing the data reported in Figure $4 \mathrm{C}$ according to eq 5 .

2). The best fit of these data shows that both the longest relaxation time and its weight decrease three-fold, while the shortest relaxation time remains unchanged over the $I_{\text {pump }}$ range explored. The average relaxation time $\langle\tau\rangle=f_{1} \tau_{1}+f_{2} \tau_{2}$ is also markedly dependent on the pump intensity, as shown in the inset of Figure 4B. These findings show that the time response of the fluorescence enhancement can be tuned by selecting the pump beam intensity.

The photobleaching induced by the pump or probe beams is weak under the present excitation conditions. Only a slight time decrease of the fluorescence signal is observed on each probe excitation pulse in the fluorescence traces (Figure 3A, upper curve), with a hint of molecular photobleaching from the $\mathrm{S}_{2}$ state (as confirmed by the experiments on gels described below). It is however not possible to evaluate the photobleaching rate from $\mathrm{S}_{2}$ from the solution experiments. However, the data indicate that the trend of $G(0, \omega)$ as a function of $\omega$ is not markedly affected by this minor photobleaching component. In fact, we do not observe a decrease of the average fluorescence with time under pulsed excitation, and $G(0, \omega)$ either stays constant as a function of the excitation intensity (Figure 4A) or increases (Figure 4B). A detailed prediction of the effect of photobleaching on $G(0, \omega)$ is not easy, ${ }^{44}$ and the effect of photobleaching on the modulated excitation can be more directly assessed by means of experiments in gels (see Experiments in Gels section).

Effect of the Solution Physical-Chemical Parameters on the Dynamics. Figure 4C reports the demodulation factor, $G(0, \omega)$, as a function of the modulation frequency of the probe beam under constant pump excitation $I_{\text {pump }}=1.7 \mathrm{~kW} / \mathrm{cm}^{2}$, in a wide range of $\mathrm{pH}$ values. The data, analyzed according to eq 4 , provide values of the relaxation times that increase when decreasing the solution $\mathrm{pH}$, as reported in Table 3. The average relaxation time, which changes from $\langle\tau\rangle \cong 10$ to $40 \mathrm{~ms}$ between 


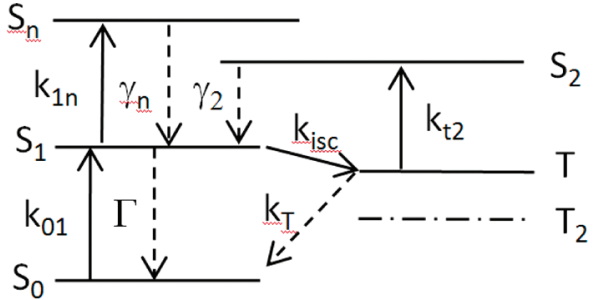

Figure 5. Energy diagram of a four-level model that accounts for the observed fluorescence enhancement properties of Mut2Q. The excitation rates $k_{1 n}, k_{01}$, and $k_{\mathrm{t} 2}$ depend on the excitation intensity; $k_{10}=\sigma_{1} I_{\mathrm{pump}}$, $k_{1 n}=\sigma_{n} I_{\text {pump }}$, and $k_{\mathrm{t} 2}=\sigma_{2} I_{\text {probe }} . \Gamma$ is the sum of the radiative, the internal conversion, and the stimulated emission rates, and the latter can be neglected in the present case, $\Gamma=\gamma_{\mathrm{R}}+k_{\mathrm{ic}}+k_{01} \cong \gamma_{\mathrm{R}}+k_{\mathrm{ic}}$. Regarding the triplet states, the intersystem crossing, $k_{\text {isc }}$, and the triplet, $k_{\mathrm{T}}$, rates account for the equilibrium between the ground and excited singlet states and the triplet trap state, T. The triplet state $T_{2}$ is a different triplet state that may account for the 10-100 $\mu$ s dynamic observed in the FCS ACFs, and its location in the energy diagram is only indicative. The spontaneous decay rates, $\gamma_{2}$ and $\gamma_{n}$, account for the decay from the $\mathrm{S}_{2}$ and $\mathrm{S}_{n}$ states.

pH 9.0 and 6.2, can be fitted to a protonation curve (inset in Figure 4C) with $\mathrm{p} K_{\mathrm{a}}=7.0 \pm 0.1$. Therefore, the enhancement dynamics contains a protonable step with a $\mathrm{p} K_{\mathrm{a}}$ value higher than that controlling the overall fluorescence emission $\left(\mathrm{p} K_{\mathrm{a}}=\right.$ $6.0 \pm 0.1)^{14}$ and closer to that found from the analysis of the fluorescence enhancement amplitude, $\Delta F / F_{0}\left(\mathrm{p} K_{\mathrm{a}}=6.5 \pm 0.1\right)$.

The enhancement dynamics is not affected appreciably by the solution viscosity up to $6 \mathrm{cPoise}$ (Figure 4D, inset) and by temperatures between 24 and $38^{\circ} \mathrm{C}$, as shown in Figure 4D for different pump values.

Energy Diagram Model. The data indicate that the first excited anionic state, $S_{1}$, is critically involved in the fluorescence enhancement effect. The decrease of the fluorescence enhancement observed at increasing pump beam intensity (Figure 1D) suggests additionally that some higher energy state $S_{n}$ needs to be included in the energy diagram. Other energy levels should also be taken into account in this scheme based on the presence of a $1-20 \mu$ s relaxation in the FCS ACFs, visible mostly at high excitation intensity and due to an equilibrium between the $\mathrm{S}_{1}$ and some triplet state. ${ }^{14,15}$ Finally, two other energy levels should account for the intensity- and $\mathrm{pH}$-dependent relaxation components observed in the 50-300 $\mu$ s range in the FCS ACFs. ${ }^{14}$

We assume that some of these pathways are decoupled since they are related to widely different time scales, and we focus here only on those energy levels which appear to be necessary to rationalize the fluorescence enhancement effect and its dynamics. As sketched in Figure 5 a trap state, T, can be populated from the first singlet excited state, $S_{1}$. We assume that molecules in the trap state can be excited by the probe beam to a state, $\mathrm{S}_{2}$, which lies approximately $3 \mathrm{eV}$ (corresponding to $420 \mathrm{~nm}$ ) above T. A direct decay from $S_{2}$ to $S_{1}$ brings then the molecule back to the fluorescence excitation-emission loop related to the transition $S_{0} \rightarrow S_{1}$. An alternative nonradiative deactivation route is from $\mathrm{T}$ to $\mathrm{S}_{0}$ with a rate $k_{\mathrm{T}}$. The state $\mathrm{T}$ is likely to be a triplet state distinct from the triplet state that is responsible for the $10-100 \mu$ s photodynamics observed in the FCS ACFs ${ }^{14,15}$ and indicated in Figure 5 as $T_{2}$ due to the wide difference of this photodynamics with respect to the millisecond enhancement activation dynamics. The excitation and emission rate constants of such a system and the corresponding cross sections, $\sigma_{1}, \sigma_{n}$, and $\sigma_{2}$, are related by $k_{10}=\sigma_{1} I_{\text {pump }}, k_{1 n}=\sigma_{n} I_{\text {pump }}$, and $k_{\mathrm{t} 2}=\sigma_{2} I_{\text {probe }}$. The relaxation rates for the various transitions are defined in the caption of Figure 5 together with the scheme of the energy diagram, whose dynamics is described by the set of linear equations

$$
\left\{\begin{array}{l}
\frac{\mathrm{dS}_{0}}{\mathrm{~d} t}=-k_{01} \mathrm{~S}_{0}+\Gamma \mathrm{S}_{1}+k_{\mathrm{T}} \mathrm{T} \\
\frac{\mathrm{d} \mathrm{S}_{1}}{\mathrm{~d} t}=k_{01} \mathrm{~S}_{0}-\left(\Gamma+k_{\mathrm{isc}}+k_{1 n}\right) \mathrm{S}_{1}+\gamma_{2} \mathrm{~S}_{2}+\gamma_{n} \mathrm{~S}_{n} \\
\frac{\mathrm{d} \mathrm{S}_{n}}{\mathrm{~d} t}=k_{1 n} \mathrm{~S}_{1}-\gamma_{n} \mathrm{~S}_{n} \\
\frac{\mathrm{dS}}{\mathrm{d} t}=k_{\mathrm{t} 2} \mathrm{~T}-\gamma_{2} \mathrm{~S}_{2} \\
\frac{\mathrm{dT}}{\mathrm{d} t}=k_{\mathrm{isc}} \mathrm{S}_{1}-\left(k_{\mathrm{T}}+k_{\mathrm{t} 2}\right) \mathrm{T}
\end{array}\right.
$$

From these equations, we can obtain the equilibrium and the dynamic response of the chromophore to the pump and the probe beams, which are analyzed separately hereafter.

It must be noticed that this energy diagram model is probably not the only one that would allow one to describe the observed trend of the fluorescence enhancement. Our choice is motivated by a minimum complexity requirement. Moreover, the mechanism just outlined is quite common in many organic dyes; ${ }^{45}$ it is known as (photoinduced) reverse intersystem crossing, and it has been investigated by means of two-color spectroscopy for cyanine dyes, ${ }^{46-48}$ for rose bengale, ${ }^{49}$ for sulfo-rhodamine $101,{ }^{50}$ and also for fluorescent proteins. ${ }^{51}$ These studies have also been performed in solutions by $\mathrm{FCS}^{47}$ or at the singlemolecule level on immobilized dyes. ${ }^{48,50} \mathrm{We}$ consider this wide series of studies a reasonable motivation to adopt reverse intersystem crossing to develop an energy diagram model as described in the following.

The functional form of the average fluorescence signal and its dependence on the pump (through $k_{01}$ ) and probe intensities (through $k_{\mathrm{t} 2}$ ), derived from the stationary solutions of eq 7 (see $\mathrm{SI})$ with the constraint that $\mathrm{S}_{0}+\mathrm{S}_{1}+\mathrm{S}_{2}+\mathrm{S}_{n}+\mathrm{T}=\langle N\rangle$ is

$$
\begin{aligned}
& \langle F\rangle\left(I_{\text {pump }}, I_{\text {probe }}\right)=\eta \gamma_{\mathrm{R}} \mathrm{S}_{1}= \\
& \eta \gamma_{\mathrm{R}} \alpha\langle N\rangle I_{\text {pump }} \\
& 1+\chi g\left(\xi I_{\text {probe }}\right)+\alpha I_{\text {pump }}\left[\psi+\frac{\beta}{\xi} g\left(\xi I_{\text {probe }}\right)+\varepsilon I_{\text {pump }}\right]
\end{aligned}
$$

In the previous equation, $\langle N\rangle$ is the average number of molecules in the observation volume, $g(x)=x /(1+x), \eta$ is the setup collection efficiency, $\gamma_{\mathrm{R}}$ is the radiative rate, $\psi=1+\left(k_{\mathrm{isc}} / k_{\mathrm{T}}\right)$, $\varepsilon=\left(\sigma_{n} / \gamma_{n}\right), \alpha=\sigma_{1} \tau, \xi=\left(\sigma_{2} / k_{\mathrm{T}}\right), \tau=1 / \gamma_{\text {tot }}$ is the $S_{1}$ state lifetime, $\beta=\left(k_{\text {isc }} / k_{\mathrm{T}}\right) \sigma_{2}\left(\left(1 / \gamma_{2}\right)-\left(1 / k_{\mathrm{T}}\right)\right)$, and $\chi=k_{\mathrm{isc}} / \gamma_{\text {tot }}$, which is assumed to be negligible in the following analysis.

We analyze now the data collected as a function of the pump and the probe intensities under continuous wave excitation in terms of eq 8 . To this purpose, it is convenient to specialize eq 8 to simplified expressions. When analyzing the dependence on the pump excitation intensity, under no probe beam excitation, we can write 


$$
\left.\langle F\rangle\left(I_{\text {pump }}\right)\right|_{I_{\text {probe }}}=\frac{A I_{\text {pump }}}{1+B I_{\text {pump }}\left[1+C I_{\text {pump }}\right]} \quad \text { with }\left\{\begin{array}{l}
A=\eta\langle N\rangle \sigma_{1} \phi_{1} \\
B=\sigma_{1} \tau \psi \\
C=\frac{\varepsilon}{\psi}
\end{array}\right.
$$

Instead, when analyzing the trend of the fluorescence output as a function of the probe intensity under constant pump intensity, we can write

$$
\left.\langle F\rangle\left(I_{\text {probe }}\right)\right|_{I_{\text {pump }}}=\frac{D}{1+\frac{E}{\xi} g\left(\xi I_{\text {probe }}\right)} \quad\left\{\begin{array}{c}
D=\eta\langle N\rangle \frac{\sigma_{1} \phi I_{\text {pump }}\left(1+\chi g\left(\xi I_{\text {probe }}\right)\right)^{-1}}{1+\frac{\alpha I_{\text {pump }}}{1+\chi g\left(\xi I_{\text {probe }}\right)}\left[\psi+\varepsilon I_{\text {pump }}\right]} \\
E=\frac{\alpha \beta I_{\text {pump }}\left(1+\chi g\left(\xi I_{\text {probe }}\right)\right)^{-1}}{1+\frac{\alpha I_{\text {pump }}}{1+\chi g\left(\xi I_{\text {probe }}\right)}\left[\psi+\varepsilon I_{\text {pump }}\right]}
\end{array}\right.
$$

The fit of the fluorescence rate versus the pump intensity (Figure 1B) to eq 9 in the absence of the probe beam provides the best-fit values $\eta \sigma_{1}\langle N\rangle \gamma_{\mathrm{R}} \tau=\eta \sigma_{1}\langle N\rangle \phi_{1}=(1.1 \pm 0.03) \times 10^{-17} \mathrm{~cm}^{2}, \sigma_{1} \tau \psi=(7.3 \pm 0.7) \times 10^{-24} \mathrm{~cm}^{2} \mathrm{~s}$, and $\varepsilon / \psi=(4 \pm 1) \times 10^{-25} \mathrm{~cm}^{2} \mathrm{~s}$. In order to obtain a direct estimate of the relevant photophysical parameters, we must assume values for $\eta \cong 0.01, \tau \cong 1 \mathrm{~ns},{ }^{14}$ and $\phi_{1} \cong 0.25$, as found for the S65G/E222Q GFP mutant by Jung et al., ${ }^{27}$ and $\langle N\rangle \cong 40$ as measured through FCS analysis (see SI). In summary, we estimate by the above analysis the values $\sigma_{1} \cong 10^{-16} \mathrm{~cm}^{2},\left(k_{\text {isc }} / k_{\mathrm{T}}\right) \cong 70$, and $\varepsilon=\left(\sigma_{n} / \gamma_{n}\right) \cong 2.8 \times 10^{-23} \mathrm{~cm}^{2} \mathrm{~s}$. The value obtained for the $S_{0}-S_{1}$ cross section is in reasonable agreement with literature data regarding other GFP mutants. ${ }^{32}$

Regarding the effect of the probe excitation on the trend of the fluorescence emission, the data reported in Figure $1 \mathrm{C}$ have been fit to eq 10 (or eq $\mathrm{S}_{1} 9$ given in the SI) by keeping $\xi$ as a global fit parameter and finding $\xi=(2.6 \pm 0.2) \times 10^{-21} \mathrm{~cm}^{2} \mathrm{~s}$. The most relevant result is that the best-fit $\beta$ parameter (obtained from the fit of the $E$ parameter versus $I_{\text {pump }}$ in eq 10) is negative, $\beta \cong-7$ $\times 10^{-21} \mathrm{~cm}^{2} \mathrm{~s}\left(\right.$ SI Table $\mathrm{S}_{1}$, Figure $\mathrm{S}_{1}$ ), indicating that $k_{\mathrm{T}}<\gamma_{2}$ and further implying that the transition occurs mainly in the direction $\mathrm{S}_{1} \rightarrow \mathrm{T} \rightarrow \mathrm{S}_{2} \rightarrow \mathrm{S}_{1}$. The value of the parameter $\varepsilon=\left(\sigma_{n} / \gamma_{n}\right)$, obtained from the fit of $\langle F\rangle$ as a function of $I_{\text {probe }}$, is however 1 order of magnitude larger $\left(\varepsilon \cong 10^{-22} \mathrm{~cm}^{2} \mathrm{~s}\right)$ than that obtained from the fit of the data reported in Figure 1B.

The substantial consistency of the analyses of the trends of $\langle F\rangle$ as a function of the $I_{\text {pump }}$ and $I_{\text {probe }}$ intensities is further supported by the analysis of the trend of the relative enhancement amplitude, $\Delta F / F_{0}$, as a function of the pump intensity (Figure $1 D$ ). Equation 10 predicts an initial increase of this parameter followed by a decrease at large pump excitation intensities, according to the following expression

$$
\frac{\Delta F}{F_{0}} \cong-\frac{\alpha I_{\text {pump }} \frac{\beta}{\xi}}{1+\alpha I_{\text {pump }}\left[\psi+\frac{\beta}{\xi}+\varepsilon I_{\text {pump }}\right]}
$$

The decrease of $\Delta F / F_{0}$, observed at high intensities, is due to the denominator of eq 11 , whose leading term, $\cong \alpha \varepsilon I_{\text {pump }}^{2}$, is related to transitions to higher-energy states through the $\varepsilon$ parameter. The solid line reported in Figure 1D corresponds to the best fit obtained by keeping $\sigma_{1} \cong 10^{-16} \mathrm{~cm}^{2}$, and that leads to the estimate of $\varepsilon \cong 7 \times 10^{-22} \mathrm{~cm}^{2} \mathrm{~s}$ and $\beta \cong-3 \times 10^{-20} \mathrm{~cm}^{2} \mathrm{~s}$. The $\beta$ value agrees with the estimates obtained by fitting the pump intensity dependence of $\langle F\rangle$. The still larger value of $\varepsilon$ found here indicates that the enhancement is largely limited by the fluorescence saturation due to higher-energy-level transitions and suggests the possibility that the simple energy diagram proposed in Figure 5 may lack transitions from the trap state to some higher-energy states, which eventually do not lead to the $S_{1} \rightarrow T \rightarrow S_{2} \rightarrow S_{1}$ cycle. In summary, the above analysis of the trend of the fluorescence rate as a function of both the probe and the pump beam excitation intensities substantially agrees with the data presented in the literature in terms of the $S_{0}-S_{1}$ transition cross section and, most importantly, provides information on the higher-energy transitions and on the possible source of the enhancement effect in terms of the balancing between the $T \rightarrow S_{0}$ and $S_{2} \rightarrow S_{1}$ transition rates.

Regarding the enhancement photodynamics, our model should reproduce the following experimental observations: (a) the slow relaxing enhancement dynamics is found only under a modulated probe beam, (b) two major relaxation components can be detected, and (c) only one of these relaxation times depends on the pump intensity (Figure 4 and Table 2). The derivation of the relaxation times of the fluorescence signal under modulated excitation is rather involved and is reported in detail in the SI. In summary, the relaxation dynamics can be obtained by computing first the Green function of the dynamic equations (eq 7) and then convoluting these with the excitation time profile. The Green function of the dynamic system is a linear composition of exponential decays whose relaxation rates are the eigenvalues of eq 7. In order to simplify the mathematical treatment, the eigenvalues and eigenvectors analysis of eq 7 has been performed to the first-order approximation in $I_{\text {pump }}$ and $I_{\text {probe }}$, as detailed in the SI. We summarize in the following the results of the theoretical analysis for the pump and the probe modulation separately.

When the pump beam is modulated, $\mathrm{S}_{1}(t)$ depends only on the first two eigenvalues (see SI), leading to a fluorescence following instantaneously any modulation in the $\mathrm{Hz}-\mathrm{kHz}$ range, as observed in Figure $3 \mathrm{D}$ and inset. A different situation is found when analyzing the effect of the probe beam modulation on $G(0, \omega)$, as detailed in the SI. The resulting four eigenvalues are, to the first order in $\left\langle k_{\mathrm{t} 2}\right\rangle$ (or $I_{\text {probe }}$ ) and $\left\langle k_{01}\right\rangle$ (or $\mathrm{I}_{\text {pump }}$ ) 
$\left\{\begin{array}{c}\lambda_{0}=0 \\ \lambda_{1,2}=-\frac{\left(\Gamma+k_{\mathrm{isc}}+\left\langle k_{01}\right\rangle\right)}{2}\left[1 \pm \sqrt{1-\frac{4\left\langle k_{01}\right\rangle k_{\mathrm{isc}}}{\left(\Gamma+k_{\mathrm{isc}}+\left\langle k_{01}\right\rangle\right)^{2}}}\right]+\Delta_{1,2}\left(\left\langle k_{\mathrm{t} 2}\right\rangle\right) \\ \lambda_{3}=-\gamma_{2}+\Delta_{3}\left(\left\langle k_{\mathrm{t} 2}\right\rangle\right)\end{array}\right.$

The eigenvector analysis indicates that in this case, all of the four eigenvalues contribute to the $S_{1}$ decay. As in the case of the $I_{\text {pump }}$ response analysis, the first of these rates $\left(\lambda_{0}\right)$ corresponds to the equilibrium state, and the second $\left(\lambda_{1}\right)$ accounts for the fluorescence antibunching. The other two components $\left(\lambda_{2}, \lambda_{3}\right)$ correspond to the characteristic times $\tau_{1}$ and $\tau_{2}$

$$
\left\{\begin{array}{c}
\tau_{1}\left(\left\langle k_{01}\right\rangle\right)=-\frac{1}{\lambda_{2}} \cong\left\{f\left(\left\langle k_{01}\right\rangle\right)+\frac{k_{\text {isc }} k_{\mathrm{T}}\left\langle k_{01}\right\rangle}{\left[2 f\left(\left\langle k_{01}\right\rangle\right)-\Gamma-k_{\text {isc }}-\left\langle k_{01}\right\rangle\right]\left[k_{\mathrm{T}}-f\left(\left\langle k_{01}\right\rangle\right)\right]}\right\}^{-1} \\
\quad \text { with } f\left(\left\langle k_{01}\right\rangle\right)=\frac{k_{\text {isc }}\left\langle k_{01}\right\rangle}{\Gamma+k_{\text {isc }}+\left\langle k_{01}\right\rangle} \\
\tau_{2}=-\frac{1}{\lambda_{3}}=\frac{1}{\gamma_{2}}
\end{array}\right.
$$

This result is in agreement with the second basic dynamic observation, which is reported in Figures 3 and 4. In particular, the fit of the function $\tau_{1}\left(\left\langle k_{01}\right\rangle\right)$ to the data in Figure $4 \mathrm{~B}$ (inset) gives the best-fit parameters $\Gamma+k_{\text {isc }}=1.4 \pm 0.5 \times 10^{9} \mathrm{~Hz}$, $k_{\text {isc }}=450 \pm 60 \mathrm{~Hz}$, and $k_{\mathrm{T}}=8.0 \pm 0.6 \mathrm{~Hz}$, when assuming $\sigma_{1}$ $\cong 10^{-16} \mathrm{~cm}^{2}$, as found previously. This indicates that $\Gamma \cong 10^{9}$ $\mathrm{Hz}$, in agreement with the excited-state lifetime of the Mut2Q chromophore $\tau \cong 1 \mathrm{~ns} .{ }^{14}$ Moreover, the $k_{\text {isc }}$ and $k_{\mathrm{T}}$ values found here agree well with the estimate of the parameter $\psi=1+$ $\left(k_{\text {isc }} / k_{\mathrm{T}}\right) \cong 70$ obtained from the analysis of the average emission as a function of the excitation intensities. Starting from the previous estimate of $\xi=(2.6 \pm 0.2) \times 10^{-21} \mathrm{~cm}^{2} \mathrm{~s}$, we compute a cross section value, $\sigma_{2} \cong 2 \times 10^{-20} \mathrm{~cm}^{2}$, for the transition involved in the enhancement of the fluorescence, which, for $I_{\text {probe }} \cong 1 \mathrm{~kW} / \mathrm{cm}^{2}$, would imply a $\mathrm{T} \rightarrow \mathrm{S}_{2}$ excitation rate of $\cong 50 \mathrm{~Hz}$, compatible with the average relaxation time measured in Figures 3 and 4. Finally, we notice that the low value of $k_{\text {isc }}$ additionally supports our hypothesis that the trap $\mathrm{T}$ state is not the same triplet state responsible of the $10-100 \mu$ s photodynamics observed in the ACFs.

The transition rates related to the enhancement dynamics may be affected by the diffusion of the Mut2Q proteins through the observation volume. In the setup conditions used here, we observe, on average, 40 molecules diffusing through the observation volume in approximately $1.6 \mathrm{~ms}$, a time smaller than most of the light modulation times $\tau=1 / v(1-100 \mathrm{~ms})$ used in the experiments reported below. The photochromicity kinetics depends directly on the population of the $S_{0}, S_{1}, T$ and $\mathrm{S}_{2}$ states. The $\mathrm{S}_{0}$ and $\mathrm{S}_{1}$ state populations change on time scales much faster $(1-10 \mathrm{~ns})$ than the one on which the excitation light is modulated $(1-100 \mathrm{~ms})$. The $\mathrm{T}$ state populates with a $k_{\text {isc }}$ rate that corresponds to a relaxation time of about $2 \mathrm{~ms}$, on the same order of the protein diffusion time. The $S_{2}$ states becomes populated with rates on the order of $100 \mathrm{~Hz}$ or relaxation times on the order of $10 \mathrm{~ms}$, depending on the probe beam excitation intensity. To first approximation, we can then consider the $\mathrm{S}_{0}, \mathrm{~S}_{1}$ population as effectively stationary on the time scale of the probe light modulation times (see SI for additional mathematical treatment). However, for the slowest modulation frequencies $(<10 \mathrm{~Hz})$, some of the proteins may be actually diffusing out of the observation volume before they suffer a $\mathrm{T} \rightarrow \mathrm{S}_{2}$ transition entering the photochromicity transition loop. A slight systematic overestimation of the fluorescence enhancement relaxation times $\left(\tau_{1, \mathrm{R}}\right.$ and $\tau_{2, \mathrm{R}}$ in Tables 2 and 3$)$ may then be expected.

The complex photoactivation of the Mut2Q mutant differs from that of other photoactivable proteins, such as PA-GFP ${ }^{9}$ and DRONPA. ${ }^{18}$ For DRONPA, once the chromophore has undergone photoswitching from the bright anionic to the dim neutral state under the action of $490 \mathrm{~nm}$ radiation, it can be brought back to the bright state by even a single scan at 405 $\mathrm{nm}$. This cycle can be repeated several times even at the singlemolecule level. ${ }^{18}$ The emission is therefore almost completely switched off by $490 \mathrm{~nm}$ photoswitching to the protonated form, where the protein lies until it is shined by a second, more energetic laser beam. PA-GFP shows enhancement ${ }^{9}$ of the fluorescence primed by $490 \mathrm{~nm}$ light under the action of more energetic photons. The increase in the fluorescence emission is much larger than that observed here for Mut2Q and, most important, can last for days. On the contrary, Mut2Q does show a fluorescence enhancement which does not seem to be related to the neutral-anionic transition and which lasts only as long as the protein is shined by the more energetic beam, being activated in $1-10 \mathrm{~ms}$.

Experiments in Gels. We have computed the transition rates within the adopted energy model from the analysis of the FCS ACFs of proteins diffusing through the observation volume. However, our main reason for characterizing Mut2Q photochromicity was to develop methods for dynamic measurements in situations in which the mobility of the mutant protein is much lower, such as, for example, in cells. The translational diffusion coefficient of GFP changes from about $D \cong 90 \mu \mathrm{m}^{2} / \mathrm{s}$ in buffer solution to $\mathrm{D} \cong 0.1-10 \mu \mathrm{m}^{2} / \mathrm{s}$ in cells $\mathrm{s}^{52,53}$ due to the interaction with the cytosolic matrix and to its possible link to receptors. ${ }^{53,54}$ Therefore, we present here also a characterization of the photochromicity kinetics of Mut2Q in less mobile environments, such as gels. The study of possible applications of Mut2Q photochromicity in intracellular environments will be the subject of a forthcoming study.

These experiments on proteins in gels have been performed on a homemade confocal setup in which the sample can be raster-scanned by the laser beams by driving a piezo actuator. The motion of the actuator is driven by the same clock that drives the electronic shutter that determines the modulation of the probe beam. In this way, we could acquire images of the gels while modulating the probe beam in phase with the raster scanning motion. A typical result of such experiments is reported in Figure 6 (images). In this first series of experiments, the pixel size was $100 \mathrm{~nm}$, approximately half of the size of the point spread function, and the pixel dwell time was $5 \mathrm{~ms}$. The raster scanning and the modulation were phased in such a way to obtain a vertical pattern; the bright stripes correspond to the onset of the probe beam excitation, and the pump beam at 488 $\mathrm{nm}$ was always impinging on the sample. The bright-dark and dark-bright transitions were affected by a fast spatial decay $(300 \pm 100 \mathrm{~nm})$ along the scanned line ( $x$-axis in the image), which is due to the oversampling of the point spread function.

The analysis of the stripes contrast, $\Delta F / F_{0}$, as a function of the probe beam intensity under $I_{\text {pump }}=0.6 \mathrm{~kW} / \mathrm{cm}^{2}$ is reported in Figure 6 as a function of the probe intensity. The analysis of this trend by means of eq 6 provides an estimate of the saturation intensity $I_{\text {sat }}=0.33 \pm 0.1 \mathrm{~kW} / \mathrm{cm}^{2}$ in excellent agreement with the solution data reported in Table 1 . The fluorescence enhancement induced on Mut2Q by the $420 \mathrm{~nm}$ probe beam can therefore be exploited also when the protein diffusion is largely hindered. The minor photobleaching effect induced by the probe beam does not seem to hamper the exploitation of the 


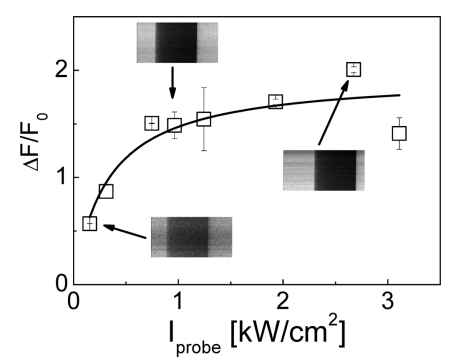

Figure 6. Experiments on Mut2Q proteins in acrylamide gels. Amplitude of the fluorescence enhancement, $\Delta F / F_{0}$, obtained on Mut2Q loaded acrylamide gels $(\mathrm{pH}=8)$ as a function of the probe intensity $(\lambda=420 \mathrm{~nm}), I_{\text {probe }}$, under the continuous action of the pump beam $\left(I_{\text {pump }}=0.6 \mathrm{~kW} / \mathrm{cm}^{2}\right)$. The values have been obtained from the analysis of the images of the gels taken under modulated probe excitation $\left(f_{\mathrm{S}}=\right.$ $1 \mathrm{~Hz}$ ). The images were acquired at $200 \times 100$ pixels with a pixel dwell time of $5 \mathrm{~ms}$. Some sample images are reported as insets. Each point of the plot is the result of the average of the relative enhancement computed over all of the rows of the image; the error bars represent the standard deviation. The solid line is the best fit of the data to eq 6 .

fluorescence enhancement in these environments, as also outlined by a second series of experiments on gels devised to measure the photochromic relaxation time $\tau_{\mathrm{R}}$.

This second series of experiments was performed on Mut2Q proteins trapped in acrylamide gels by means of an imaging protocol devised to measure the enhancement kinetics with pixel resolution. The protocol that we test here on a line scanning is based on a slight difference between the modulation wave $\left(f_{\mathrm{S}}\right)$ and the line scanning $\left(f_{\mathrm{L}}\right)$ frequencies. A second fundamental assumption is that the sample is stationary, in the sense that all of the sequentially performed line scannings are equivalent to each other. This is a critical assumption that will set a limitation to the possibility of following a time evolution of the fluorescence enhancement, as discussed later in the text. As will be clear from the analysis presented below, the number of sequential line scannings that we require to be equivalent is on the order of 10 or less.

In order to provide a simple example of this protocol, which we call here beating mode (BM) imaging, we have acquired images while keeping the " $y$-axis" of the piezo actuator drivers set to the ground level. In this case, as shown in Figure 7, the image is a collection of lines each of which describes a square wave with a phase that increases linearly along the $y$-axis. Altogether, the image gets the form of a wave propagating in the $\mathbf{u}$ direction (see Figure 7A) that forms an angle with the $y$-axis whose value depends on the frequency mismatch. In the example reported in Figure 7A, the line scanning and the shutter modulation (square wave) frequencies were $f_{\mathrm{L}}=1.9608 \mathrm{~Hz}$ and $f_{\mathrm{S}}=9.78 \mathrm{~Hz}$, respectively. From the pixel dwell time, set here to $\tau=5 \mathrm{~ms}$, and from the number of pixels per row (100), we would expect a line scanning frequency of $f_{\mathrm{L}}=2 \mathrm{~Hz}$. The slight difference between this and the actual value, $f_{\mathrm{L}}$, is due to dead time needed to position the piezo system at the beginning of each line scan. We call hereafter BM images those images acquired with this protocol.

We can scan any BM image in three different directions, the $x$-axis, the $y$-axis and an axis $v$ perpendicular to the $\mathbf{u}$ vector. These axes are endowed with different effective time scales. Along the $x$-axis, the time scale is determined by the pixel dwell time, in this case, $5 \mathrm{~ms}$. Along the $v$-axis, we can compute the time step per line from the line scanning frequency, in this case, $1.9608 \mathrm{~Hz}$, which corresponds to $\tau_{v} \cong 0.510 \mathrm{~s}$. Along the $y$-axis, the time scale, $\tau_{y}$, is set by the difference in frequency between the line scanning and the shutter opening, as sketched in Figure 7 A. The value of $\tau_{y}$ can be computed from the measured number of rows, $M$, after which a full modulation period has been scanned ( $M \cong 83$ rows in Figure 7 ) along the $y$-axis and from the modulation frequency of the electronic shutter (and therefore of the probe beam) as $\tau_{y}=\left(f_{\mathrm{S}} M\right)^{-1}$. In the case reported in Figure $7, f_{\mathrm{S}}=9.78 \mathrm{~Hz}, M \cong 83$, and $\tau_{y} \cong 1.23 \mathrm{~ms}$.

The image profile along the $v$-axis provides us with information on the amount of photobleaching induced by either the pump only (dark bands) or the pump\&probe excitation (bright bands) during this type of scanning. The plots reported in Figure $7 \mathrm{~B}$ indicate for the present case $\left(I_{\text {probe }} \cong 0.15 \mathrm{~kW} / \mathrm{cm}^{2}, I_{\text {pump }} \cong\right.$ $10 \mathrm{~kW} / \mathrm{cm}^{2}$ ) decay times of $44 \pm 3$ and $43 \pm 2.5 \mathrm{~s}$ for the pump\&probe (bright skew stripes) and the pump (dark skew stripes) only case, respectively. However, it must be noted that the bleaching time measured here on the image is an effective value not directly related to the actual protein bleaching time. In fact, we monitor subsequent pixels along the $v$-axis, treated as equivalent, that have received increasing energy doses (of either the pump or the pump\&probe beams) interleaved by times equal to the line scanning time ( $\cong 1 \mathrm{~s})$. Moreover, each pixel switches from one (pump only) to the other (pump\&probe) irradiation mode every half of a modulation period. The measured decay is however the relevant parameter for the present image acquisition mode since it corresponds to approximately 90 scanned lines and it ensures us the possibility to perform several $(\cong 10)$ repeated scannings on each line in order to obtain the dynamic information as outlined hereafter. In the following analysis, we have therefore not taken into account the corrections of the $y$-profiles for this photobleaching effect.

The profile of BM images along the $x$-axis allows one to measure the fluorescence enhancement amplitude on each pixel. This can be accomplished by performing the spatial first-order derivative along the row, setting a threshold level on it in order to select only the pixels on which the bright-dark or dark-bright transition can be clearly discerned and by averaging pixel by pixel over a minimum set of rows that correspond to a full half modulation period, about 40 rows for the image acquisition parameters used in the example given in Figure 7. As an example of this procedure, we report in Figure 8A the $x$-axis profile of four rows taken 10 rows apart on the same image reported in Figure 7A. The absolute value of the derivative is reported in the corresponding layer (open squares) and clearly indicates the pixels on which the maximal change in the fluorescence occurs. We assume that fluorescence gain (low to high transition) is equal to the fluorescence loss (high to low transition) and use the absolute value of the derivative of the $x$-axis profile, therefore halving the number of rows needed to compute the fluorescence enhancement pixel by pixel over the whole row ( $x$-axis). The threshold value is to be chosen according to the probe and the pump excitation intensities that affect the value of $\Delta F$ and $F_{0}$; for example, reported here, we found that the value $\Delta F / F_{0}=0.5$ allowed one to clearly determine the pixels on which the maximal change $\Delta F$ occurred. The result of the pixel by pixel averaging described above is reported in Figure $8 \mathrm{~B}$ as the plot of the relative fluorescence enhancement as a function of the position along the row. This analysis can be extended to a whole image by devising a suitable scanning algorithm synchronized with the shutter driving waveform. Also, the number of line scannings needed to cover the whole set of pixels on the row can be diminished with respect to the value $(\cong 40)$ used in Figure 8 . For the acquisition parameters used in Figures 7 and 8, we could use a minimum 


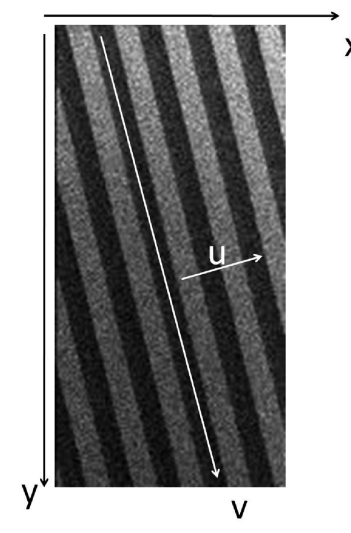

$\mathrm{x}$

(A)
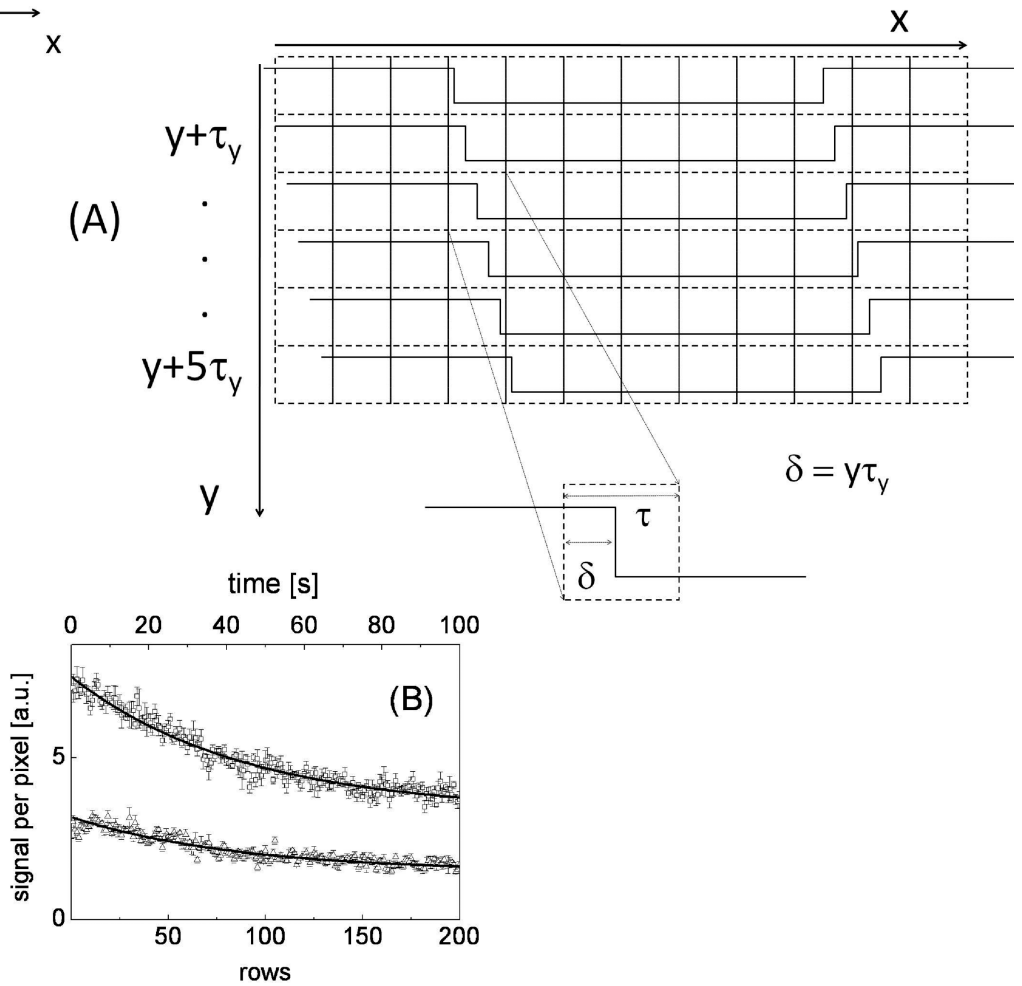

Figure 7. Acrylamide gels experiments in the beating mode. The gels were scanned under conditions in which a slight difference exists between the line scanning and the modulation wave frequencies that yields a time shift $\delta$ per subsequent row. The $y$-axis piezo control input was set to ground during the whole scanning. The "image" reported is therefore only the collection of several line scannings of the same row on the gel. (A) The image, acquired in the beating mode, was $10 \times 20 \mu \mathrm{m}^{2}(100 \times 200$ pixels $)$ in size, and it was acquired at a $f_{\mathrm{L}}=1.9608 \mathrm{~Hz}$ line scanning frequency while the shutter frequency was set at $f_{\mathrm{S}}=9.78 \mathrm{~Hz}$. The corresponding time shift per row is $\tau_{y} \cong 1.23 \mathrm{~ms}$. The $x$-, $y$-, and $v$-axes are indicated as arrowed lines. The sketch outlines the time scanning along the three axes. The blowup illustrates the role of the time shift per row, $\tau_{y}$, of the pixel dwell time, $\tau$, and of the time shift $\delta$ on each pixel. (B) Plot of the average of five profiles measured along bright (open squares) and dark (open triangles) $v$ lines on the image reported in (A). The time scale is set by the line scanning frequency. The solid lines are the best-fit exponential fit to the data (see text).

of 10 line scannings per row to build the entire profile of $\Delta F$ / $F_{0}$ over the row.

We finally discuss the analysis of the $y$-axis image profile that offers the possibility of measuring the fluorescence activation kinetics on each pixel of the row. In fact, the fluorescence collected per pixel is the integration of the photon counts detected during the pixel dwell time, $\tau$ (see Figure 7A). During a portion $\delta$ of this time stretch, the probe beam may be switched on, as sketched in Figure 7A, or off. The rise time of the probe intensity due to the finite opening time of the electronic shutter has been estimated to be less than $1 \mathrm{~ms}$. The $\delta$ time increases, in the example given in Figures 7 and 8 , in steps of $\tau_{y} \cong 1.23$ $\mathrm{ms}$. When approaching an off-on transition, the signal collected on the pixel characterized by a shift value $\delta$ is given by the integration

$$
\langle F\rangle(\delta)=\int_{0}^{\tau} \mathrm{d} t F(t)=\int_{0}^{\delta} \mathrm{d} t F_{\text {off }}(t)+\int_{\delta}^{\tau} \mathrm{d} t F_{\text {on }}(t)
$$

where $F_{\text {on }}(t)$ and $F_{\text {off }}(t)$ represent the fluorescence photon counting rates when the probe beam is on or off, respectively. When approaching an on-off transition, instead, the roles of $F_{\text {on }}$ and $F_{\text {off }}$ in eq 14 are exchanged. Referring to eq 14 , three cases can then be envisioned. When the probe beam is always on $(\delta=0)$ or off $(\delta=\tau)$ during the pixel dwell time, the fluorescence signal is simply the average of the emission under the action of the probe\&pump beams or of the pump beam alone. For those pixels, we expect to obtain fairly constant signals apart from any photobleaching effect. We call these two levels, B (bright, pump\&probe excitation) and D (dim, pump only excitation). A third case corresponds to the situation when the switching on (or off) of the probe beam occurs within the pixel dwell time duration, as outlined in the sketch of Figure 7A. In this case, if we assume an infinitely sharp transition between the $\mathrm{B}$ and $\mathrm{D}$ levels, we can compute

$$
\langle F\rangle=\frac{1}{\tau} \int_{0}^{\tau} \mathrm{d} t F(t)=\frac{\delta}{\tau} D+\frac{(\tau-\delta)}{\tau} B=B-\delta \frac{|D-B|}{\tau}
$$

for an on-off sharp transition and

$$
\langle F\rangle=\frac{1}{\tau} \int_{0}^{\tau} \mathrm{d} t F(t)=\frac{\delta}{\tau} B+\frac{(\tau-\delta)}{\tau} D=D+\delta \frac{(B-D)}{\tau}
$$

for an off-on sharp transition. The parameter $\delta$ is actually the $y$-coordinate referred to the $y$-value, $y_{0}$, that represent the onset of the transition, $\delta=y-y_{0}$. We expect therefore that the profile along the $y$-axis of a BM image resembles a square wave with linearly increasing or decreasing ramps described by eqs $15 \mathrm{a}$ and $15 \mathrm{~b}$. This is actually observed when collecting BM images under the modulation of the pump beam only on Mut2Q or fluorescein gels, as reported in Figure 9A. In this case, as shown in the blowups of Figure 9A, the on-off and off-on transitions are well-described by linear trends with slopes given by 4650 


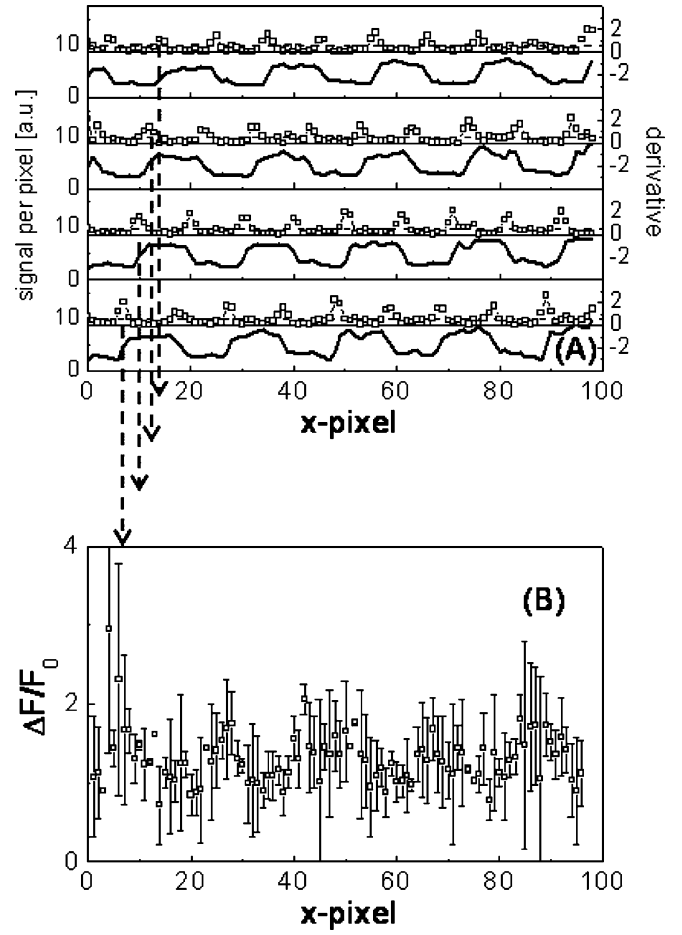

Figure 8. Computation of the fluorescence relative enhancement, $\Delta F /$ $F_{0}$, with pixel resolution. Images acquired in beating mode are analyzed along the $y$-axis, row by row. From the derivative along the $x$-axis, we can compute the $\Delta F / F_{0}$ factor. Due to the small value of the time shift per row, $\delta \cong 1.23 \mathrm{~ms}$ in the presented case, about four contiguous rows contribute to the average of the $\Delta F / F_{0}$ value on each pixel. (A) Profile of the image along the $x$-axis for the rows numbered 12, 22, 32 , and 42 (from the bottom to the top layers, thick solid lines) of the same image reported in Figure 7A. The derivatives of these profiles are reported in the same layers as open squares. (B) Value of $\Delta F / F_{0}$ computed on each pixel of the row.

\pm 30 and $4800 \pm 30 \mathrm{~Hz}$, respectively. The transitions occur indeed on about four pixels along the $y$-axis, as expected due to the choice of $\tau=5 \mathrm{~ms}$ and to the $\tau_{y}$ value $\tau_{y} \cong 1.23 \mathrm{~ms}$. Moreover, we find a quantitative agreement between the measured slopes and the computation of the values $(B-D) / \tau$ $=5500 \pm 500 \mathrm{~Hz}$ and $|D-B| / \tau=5600 \pm 800 \mathrm{~Hz}$ performed directly on the $y$-axis profile.

What happens instead when we measure a $y$-axis profile of the BM image acquired on Mut2Q loaded gels under the probe beam modulation is reported in Figure 9B. The linear increase (decrease) of the fluorescence in the off-on (on-off) transitions is rounded off, giving hints for a finite time response to the probe beam switching off. It must be noted that the shape of the on-off transitions depends on the pump intensity, as expected from the results obtained in solutions and summarized in the Table 2. However, we also notice that much less pronounced dependence is found for the off-on transitions. This behavior is not unexpected since the fluorescence enhancement is related to transitions from the $T$ to the $S_{2}$ state due to the probe beam activation. On the other hand, the off transition is determined by the repopulation of the $\mathrm{T}$ state that critically depends on the excitation rate to the $S_{1}$ state. We expect therefore that a detailed analysis of the energy diagram model, specialized to the BM acquisition mode, should account also for these observations. However, this issue is out of the main scope of the present report, which is the proof-of-concept of pixel-dependent measurements of the fluorescence enhancement amplitude and relaxation times.

The trend of the on-off and off-on transitions can be simply analyzed if we assume that the response of the protein
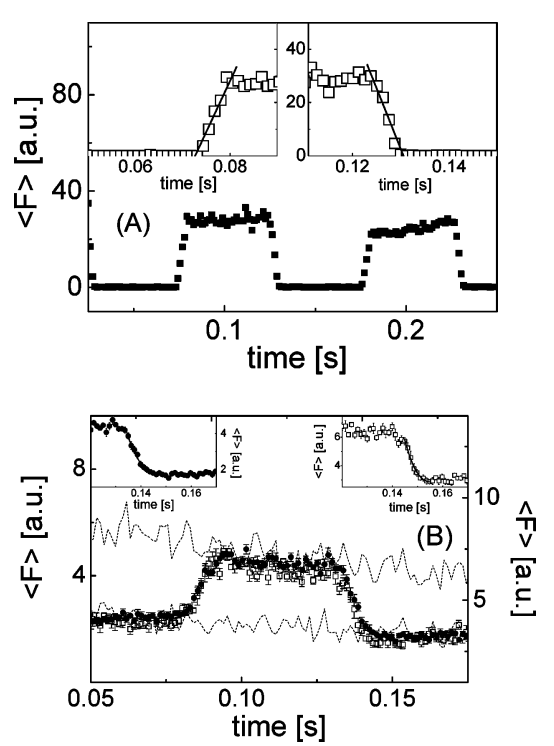

Figure 9. Evaluation of the enhancement activation time on the beating mode images. (A) Results of control experiments performed on fluorescein loaded acrylamide gels $(\mathrm{pH}=8)$. The $y$-axis profile of a BM image acquired with a line pixel dwell time of $\tau=10 \mathrm{~ms}$ and under a modulation frequency of $f_{\mathrm{S}}=9.78 \mathrm{~Hz}$ is reported in the main panel. The insets report the blowups of an off-on (left) and on-off (right) transitions together with a linear fit to the data according to eqs $15 \mathrm{a}$ and $15 \mathrm{~b}$. (B) Example of the $y$-axis profile of a BM image acquired on Mut2Q loaded acrylamide gels acquired with the same parameters as those used for the fuorescein gels. The open squares and the filled circles refer to the pump excitation intensities $I_{\text {pump }} \cong 16$ and $8 \mathrm{~kW} /$ $\mathrm{cm}^{2}$, respectively $\left(I_{\text {probe }} \cong 1.8 \mathrm{~kW} / \mathrm{cm}^{2}\right)$. The dashed curves report a $v$-axis scan along a bright (upper most) and dim (lower most) oblique stripe in the image. The insets show a blowup of the on-off transition for these two pump intensity values, together with a best-fit function according to eq 18. The best-fit parameters are $B=4.4 \pm 0.1, \Gamma=55$ $\pm 15 \mathrm{~Hz},|\mathrm{D}-\mathrm{B}| / \tau=480 \pm 90$, and $|\mathrm{D}-\mathrm{B}| /(\Gamma \tau)=17 \pm 2$ for the $I_{\text {pump }}$ $\cong 8 \mathrm{~kW} / \mathrm{cm}^{2}$ case and $B=5.9 \pm 0.2, \Gamma=88 \pm 20 \mathrm{~Hz},|\mathrm{D}-\mathrm{B}| / \tau=$ $490 \pm 100$, and $\mathrm{ID}-\mathrm{B} / /(\Gamma \tau)=13.5 \pm 4$ for the $I_{\text {pump }} \cong 16 \mathrm{~kW} / \mathrm{cm}^{2}$ case.

fluorescence to the probe intensity is described by a single exponential relaxation (with relaxation rate $\Gamma$ ). The fluorescence photon counting during the pixel integration time, $\tau$, is given by the following equation

$$
F(t)=\left\{\begin{array}{cl}
D & 0 \leq t<\delta \\
D+(B-D)(1-\exp [-\Gamma t]) & \delta \leq t \leq \tau
\end{array}\right.
$$

With the present choice of the BM images acquisition parameters, the time resolution given by $\tau_{y} \cong 1.23 \mathrm{~ms}$ does not allow one to discern two exponential relaxations as done in the solution experiments. A symmetric expression to eq 16 is given for the on-off transition. By employing eq 16 in eq 14, eqs $15 \mathrm{a}$ and $15 \mathrm{~b}$, the functional form of $\langle F\rangle$, becomes then nonlinear as a function of the $y$-axis time, $\delta$

$$
\left\{\begin{array}{l}
\langle F\rangle(\delta)=\frac{1}{\tau} \int_{0}^{\tau} \mathrm{d} t F(t)=D+\frac{(B-D)}{\tau}\left[\delta+\frac{(1-\exp [-\Gamma(\tau-\delta)])}{\Gamma}\right] \\
0 \leq \delta
\end{array}\right.
$$

for an off-on transition and 


$$
\left\{\begin{array}{l}
\langle F\rangle(\delta)=\frac{1}{\tau} \int_{0}^{\tau} \mathrm{d} t F(t)=B-\frac{|D-B|}{\tau}\left[\delta+\frac{(1-\exp [-\Gamma(\tau-\delta)])}{\Gamma}\right] \\
0 \leq \delta
\end{array}\right.
$$

for an on-off transition. Here, the value $\delta=0$ represents the time (or pixel along the $y$-axis profile) at which the probe beam is switched on or off.

Equations 17 and 18 closely describe the observed trends as illustrated in Figure 9B (insets). Actually, we should take into account also how the relaxing fluorescence signal is integrated in the pixel when the switching on or switching off of the probe beam occurs close to the pixel dwell time though not within it, that is, for $\delta<0$. This would bring us to a slightly more complex trend (reported in the SI) than that reported in eqs 17 and 18. We use here for sake of simplicity these simplified equations in order to provide a proof-of-principle of the proposed dynamic imaging method.

The best-fit values (see figure caption) of the relaxation rates are $\Gamma=55 \pm 15 \mathrm{~Hz}$ and $\Gamma=90 \pm 20 \mathrm{~Hz}$ for the pump excitation intensities $I_{\text {pump }} \cong 8$ and $16 \mathrm{~kW} / \mathrm{cm}^{2}$, respectively. The corresponding relaxation times should correspond to the $\tau_{2 \mathrm{R}}$ values reported in Table 2 . In fact, the time resolution obtained here by the beating acquisition mode, $\tau_{y} \cong 1.23 \mathrm{~ms}$, does not allow one to discriminate the contribution of the fast relaxation time, $\tau_{1 \mathrm{R}} \cong 1 \mathrm{~ms}$ (see Table 2 ), obtained in the solution experiments. The corresponding relaxation times, $\tau_{2 \mathrm{R}}$ $=1 / \Gamma$, that can be recovered from the $y$-axis profile analysis are then $\tau_{2 \mathrm{R}}=18 \pm 5$ and $11 \pm 3 \mathrm{~ms}$ for the $I_{\text {pump }} \cong 8$ and 16 $\mathrm{kW} / \mathrm{cm}^{2}$ cases, therefore in very good agreement with the solution results (see Table 2). This type of analysis can be performed on a per pixel level by analyzing all of the $y$-axis profiles that can be measured on a BM image, as shown in Figure 7. Our aim here was only to provide proof-of-concept experiments that allow one to envision the possibility of measuring on single pixels of an image both the fluorescence enhancement amplitude and its characteristic activation/ deactivation times.

\section{Conclusions}

The Mut2Q fluorescence enhancement amplitude can be exploited for modulated excitation imaging. The characterization given here of the amplitude and kinetics of the fluorescence enhancement of Mut2Q is an essential step toward future applications in modulated imaging. The marked dependence of the fluorescence enhancement and its relaxation time, $\langle\tau\rangle$, on the solution $\mathrm{pH}$ might also be exploited in the future for in vitro and intracellular measurements of $\mathrm{pH}$, an aim that was not in the scope of this report.

The four-energy-level scheme solved for the average equilibrium response and for fluorescence enhancement dynamics suggests that the observed phenomenon is related to a single feedback loop between a triplet (or long-lived) state and an excited state from which the excited singlet state can be repopulated. It is likely that also other transitions to higher energy levels from the $\mathrm{T}$ trap state are present. This theoretical description, the experimental estimate of the number and values of the relaxation times, and the tentative assignment of this dynamics to specific transitions suggest also applications of Mut2Q in modulated excitation fluorescence imaging. In fact, the enhancement factor increases rapidly with the probe beam intensity, the most effective case occurring at low pump beam intensity. This opens up the possibility of performing fluorescence imaging under modulated probe beam intensity while keeping the pump excitation at a minimum in a way similar to what was recently proposed by the Marriott group. ${ }^{13}$ A phasesensitive acquisition of the fluorescence output per pixel would then allow one to increase the image signal-to-noise ratio. Other interesting scenarios could be opened by devising new mutants in which the fluorescence activation is primed by two-photon infrared absorption and/or with activation times that fall in a shorter time window. We are currently investigating these possibilities.

An example of future fluorescence microscopy applications of Mut2Q or similar mutants has been given here by devising imaging protocols that allow the pixel-resolved measurement of the enhancement amplitude and relaxation times of the proteins trapped in gel matrixes. These experiments are thought to mimic, in terms of reduced mobility, the case of proteins in intracellular environments. We have illustrated how a simple synchronous-phase-resolved imaging method, called here beating mode imaging, allows one, in fact, to measure pixel by pixel the fluorescence enhancement and its activation/deactivation characteristic time and to have an estimate of the possible photobleaching on the immobilized proteins. Though the data reported here allow one to envision the possibility of using such imaging protocols and similar mutants for intracellular dynamic measurements, still a number of issues should be addressed in the near future. Experiments on chimeric proteins bringing Mut2Q and receptors specific for intracellular compartments are in progress in order to ascertain the potential of the proposed imaging protocols for cellular biophysical studies.

Acknowledgment. This research has been partially funded by the PRIN Projects 2006 to M.C. and S.B. (2006025255) and by the ENCITE Project (EU, FP7, Grant Agreement No: 201842) funding to G.C. We also acknowledge the financial support of Fondazione Banca del Monte for the acquisition of the CCD spectrofluorimeter.

Supporting Information Available: Further experimental and theoretical details and results. This material is available free of charge via the Internet at http://pubs.acs.org.

\section{References and Notes}

(1) Tsien, R. Y. Annu. Rev. Biochem. 1998, 67, 509-544.

(2) Chalfie, M.; Tu, Y.; Euskirchen, G.; Ward, W. W.; Prasher, D. C. Science 1994, 263, 802-805. 364.

(3) Heintzmann, R.; Gustafsson, G. L. Nat. Photonics 2009, 3, 362-

(4) Westphal, V.; Kastrup, L.; Hell, S. W. Appl. Phys. B: Laser Opt. 2003, 77, 377-380.

(5) Hofmann, M.; Eggeling, C.; Jakobs, S.; Hell, S. W. Proc. Natl. Acad. Sci. U.S.A. 2005, 102, 17565-17569. 795.

(6) Rust, M. J.; Bates, M.; Zhuang, X. W. Nat. Methods 2006, 3, 793-

(7) Hess, S. T.; Girirajan, T. P. K.; Mason, M. D. Biophys. J. 2006, 91, 4258-4272.

(8) Betzig, E.; Patterson, G. H.; Sougrat, R.; Lindwasser, O. W.; Olenych, S.; Bonifacino, J. S.; Davidson, M. W.; Lippincott-Schwartz, J.; Hess, H. F. Science 2006, 313, 1642-1645.

(9) Patterson, G. H.; Lippincott-Schwartz, J. Science 2002, 297, 18731877.

(10) Folling, J.; Belov, V.; Riedel, D.; Schoenle, A.; Egner, A.; Eggeling, C.; Bossi, M.; Hell, S. W. ChemPhysChem 2008, 9, 321-326.

(11) Lippincott-Schwartz, J.; Patterson, G. H. Trends Cell Biol. 2009, $19,555-565$.

(12) Min, W.; English, B. P.; Luo, G. B.; Cherayil, B. J.; Kou, S. C.; Xie, X. S. Acc. Chem. Res. 2005, 38, 923-931.

(13) Marriott, G.; Mao, S.; Sakata, T.; Ran, J.; Jackson, D. K.; Petchprayoon, C.; Gomez, T. J.; Warp, E.; Tulyathan, O.; Aaron, H. L.; Isacoff, E. Y.; Yan, Y. Proc. Natl. Acad. Sci. U.S.A. 2008, 105, 1778917794. 
(14) Bosisio, C.; Quercioli, V.; Collini, M.; D’Alfonso, L.; Baldini, G.; Bettati, S.; Campanini, B.; Raboni, S.; Chirico, G. J. Phys. Chem. B 2008, 112, 8806-8814.

(15) Haupts, U.; Maiti, S.; Schwille, P.; Webb, W. W. Proc. Natl. Acad. Sci. U.S.A. 1998, 95, 13573-13578.

(16) Moerner, W. E. J. Chem. Phys. 2002, 117, 10925-10937.

(17) Dickson, R. M.; Cubitt, A. B.; Tsien, R. Y.; Moerner, W. E. Nature 1997, 388, 355-358.

(18) Habuchi, S.; Ando, R.; Dedecker, P.; Verheijen, W.; Mizuno, H.; Miyawaki, A.; Hofkens, J. Proc. Natl. Acad. Sci. U.S.A. 2005, 102, 95119516.

(19) Rittweger, E.; Rankin, B. R.; Westphal, V.; Hell, S. W. Chem. Phys. Lett. 2007, 442, 483-487.

(20) Cinelli, R. A. G.; Pellegrini, V.; Ferrari, A.; Faraci, P.; Nifosi, R.; Tyagi, M.; Giacca, M.; Beltram, F. Appl. Phys. Lett. 2001, 79, 3353-3355.

(21) Jung, G.; Brauchle, C.; Zumbusch, A. J. Chem. Phys. 2001, 114, 3149-3156.

(22) Jung, G.; Wiehler, J.; Steipe, B.; Brauchle, C.; Zumbusch, A ChemPhysChem 2001, 2, 392-396.

(23) Yang, H.; Yang, H.; Luo, G.; Karnchanaphanurach, P.; Louie, T. M.; Rech, I.; Cova, S.; Xun, L.; Xie, X. S. Science 2003, 302, 262-266. (24) Wong, F. H. C.; Banks, D. S.; Abu-Arish, A.; Fradin, C. J. Am. Chem. Soc. 2007, 129, 10302-10303.

(25) Bjornberg, O.; Ostergaard, H.; Winther, J. R. Biochemistry 2006, 45, 2362-2371.

(26) Hess, S. T.; Heikal, A. A.; Webb, W. W. J. Phys. Chem. B 2004, 108, 10138-10148.

(27) Jung, G.; Wiehler, J.; Zumbusch, A. Biophys. J. 2005, 88, 19321947.

(28) Jung, G.; Zumbusch, A. Microsc. Res. Tech. 2006, 69, 175-185.

(29) Zumbusch, A.; Jung, G. Single Mol. 2000, 1, 261-270.

(30) Jung, G.; Mais, S.; Zumbusch, A.; Brauchle, C. J. Phys. Chem. A 2000, 104, 873-877.

(31) Abbruzzetti, S.; Grandi, E.; Viappiani, C.; Bologna, S.; Campanini, B.; Raboni, S.; Bettati, S.; Mozzarelli, A. J. Am. Chem. Soc. 2005, 127 626-635.

(32) Campanini, B.; Bologna, S.; Cannone, F.; Chirico, G.; Mozzarelli, A.; Bettati, S. Protein Sci. 2005, 14, 1125-1133.

(33) Chirico, G.; Cannone, F.; Beretta, S.; Diaspro, A.; Campanini, B.; Bettati, S.; Ruotolo, R.; Mozzarelli, A. Protein Sci. 2002, 11, 1152-1161.
(34) Chirico, G.; Cannone, F.; Diaspro, A. J. Phys. D: Appl. Phys. 2003, $36,1682-1688$.

(35) Cormack, B. P.; Valdivia, R. H.; Falkow, S. Gene 1996, 173, 3338.

(36) Widengren, J.; Terry, B.; Rigler, R. Chem. Phys. 1999, 249, 259271.

(37) Caccia, M.; Sironi, L.; Collini, M.; Chirico, G.; Zanoni, I.; Granucci, F. Eur. Biophys. J. Biophy 2008, 37, 979-987.

(38) Malengo, G.; Milani, R.; Cannone, F.; Krol, S.; Diaspro, A.; Chirico, G. Rev. Sci. Instrum. 2004, 75, 2746-2751.

(39) Chirico, G.; Gardella, M. Appl. Opt. 1999, 38, 2059-2067.

(40) Lakowicz, J. In Principles of Fluorescence Spectroscopy; Springer: Singapore, 2006; pp 157-204.

(41) Chattoraj, M.; King, B. A.; Bublitz, G. U.; Boxer, S. G. Proc. Natl. Acad. Sci. U.S.A. 1996, 93, 8362-8367.

(42) Eggeling, C.; Widengren, J.; Rigler, R.; Seidel, C. A. M. Anal. Chem. 1998, 70, 2651-2659.

(43) Kohl, T.; Schwille, P. Adv. Biochem. Eng./Biotechnol. 2005, 95, 107-142.

(44) Petrášek, Z.; Schwille, P. ChemPhysChem 2008, 9, 147-158.

(45) Reindl, S.; Penzkofer, A. Chem. Phys. 1996, 213, 429-438.

(46) Redmond, R. W.; Kochevar, I. E.; Krieg, M.; Smith, G.; McGimpsey, W. G. J. Phys. Chem. A 1997, 101, 2773-2777.

(47) Widengren, J.; Seidel, C. A. M. Phys. Chem. Chem. Phys. 2000, 2, 3435-3441.

(48) Huang, Z. X.; Ji, D. M.; Xia, A. D. Colloids Surf., A 2005, 25758, 203-209.

(49) Larkin, J. M.; Donaldson, W. R.; Foster, T. H.; Knox, R. S. Chem. Phys. 1999, 244, 319-330.

(50) English, D. S.; Harbron, E. J.; Barbara, P. F. J. Phys. Chem. A 2000, 104, 9057-9061.

(51) Ringemann, C.; Schoenle, A.; Giske, A.; von Middendorff, C.; Hell, S. W.; Eggeling, C. ChemPhysChem 2008, 9, 612-624.

(52) Digman, M. A.; Brown, C. M.; Sengupta, P.; Wiseman, P. W.; Horwitz, A. R.; Gratton, E. Biophys. J. 2005, 89, 1317-1327.

(53) Bizzarri, R.; Serresi, M.; Luin, S.; Beltram, F. Anal. Bioanal. Chem. 2009, 393, 1107-1122.

(54) Bizzarri, R.; Arcangeli, C.; Arosio, D.; Ricci, F.; Faraci, P.; Cardarelli, F.; Beltram, F. Biophys. J. 2006, 90, 3300-3314.

JP910075B 